\title{
Space-Time Coded Generalized Spatial Modulation for Sparse Code Division Multiple Access
}

\author{
Yusha Liu, Luping Xiang, Member, IEEE, Lie-Liang Yang, Fellow, IEEE, and Lajos Hanzo, Fellow, IEEE
}

\begin{abstract}
Space-time coded generalized spatial modulationaided sparse code division multiple access (STC/GSM-SCDMA) is proposed, which exploits the two-dimensional transmit diversity potential of both the spatial and of the frequency domain. Hence, it constitutes a promising solution for the pervasive connectivity of devices in next-generation nonorthogonal multiple access (NOMA) systems. More explicitly, our STC/GSM scheme achieves diversity in the spatial-domain, while the sparse signalspreading action of SCDMA results in frequency-domain (FD) diversity. A single-user bit error rate (BER) bound is derived as the benchmark of the BER performance of our STC/GSMSCDMA system. Furthermore, a pair of novel detectors, namely a bespoke message passing aided (MPA) detector and a tailormade approximate message passing (AMP) detector are conceived by designing a new factor graphs for our proposed STC/GSMSCDMA system. The performance of these detectors is characterized in terms of their BER vs. complexity. Our simulation results show that the proposed AMP detector is capable of operating within $2 \mathrm{~dB}$ of the MPA detector's signal-to-noise ratio (SNR) requirement, while supporting a normalized user load of $150 \%$, despite its appealing low complexity, which is about 1000 times lower than the MPA detector.
\end{abstract}

Index Terms-Space-time coding, generalized spatial modulation, sparse code multiple access, nonorthogonal multiple access

\section{INTRODUCTION}

Nonorthogonal multiple access (NOMA), a promising solution to pervasive connectivity, has found diverse applications in next-generation wireless communications scenarios, such as massive machine type communication (mMTC) [1-3], Internet of Things (IoT) [4] and unmanned aerial vehicle (UAV) communications $[5,6]$. Furthermore, the intrinsic integration of NOMA and emerging techniques, such as reconfigurable intelligent surfaces (RIS) and orthogonal time frequency space (OTFS) modulation have been investigated in $[7,8]$. In this context, both code-domain [9-11] and power-domain NOMA [12-14] techniques have found favor in compelling practical applications. While power-domain NOMA adopts superposition codes for multiplexing in the power-domain, code-domain NOMA achieves multiplexing by using nonorthogonal user-specific spreading sequences. At the receiver side, low-complexity successive interference cancellation (SIC) is employed for power-domain NOMA. By contrast,

The authors are with School of Electronics and Computer Science, University of Southampton, SO17 1BJ, UK. (E-mail: yl6g15, 1x1g15, 1ly, lh@soton.ac.uk). (Corresponding author: Luping Xiang)

L. Hanzo would like to gratefully acknowledge the financial support of the Engineering and Physical Sciences Research Council projects EP/Noo4558/1, EP/PO34284/1, COALESCE, of the Royal Society's Global Challenges Research Fund Grant as well as of the European Research Council's Advanced Fellow Grant QuantCom. the popular state-of-the-art detector of code-domain NOMA schemes, such as low density signature (LDS) [9] and sparse code multiple access (SCMA) [11] arrangements is the message passing aided (MPA) detector. The extensive research dedicated to LDS and sparse code multiple SCMA, have shown that both of them are capable of achieving near-singleuser performance even at a normalized user load of $200 \%$, by adopting MPA detection [11]. In amalgamation with fasterthan-Nyquist (FTN) signaling, FTN-SCMA investigated in [15-17] was shown to further enhance the spectral efficiency of SCMA systems. This impressive performance demonstrates their potential in heavily-loaded mMTC networks.

However, the extra interference imposed by code-domain NOMA can only be mitigated at an increased receiver complexity. For the sake of counter-balancing this complexity escalation, spatial modulation (SM) [18] has been proposed for reducing the number of radio frequency (RF) chains of multiple-input multiple-output (MIMO) systems, hence mitigating the receiver complexity. By adopting conventional SM or generalized SM (GSM) [19], only a single or a sub-group of the transmit antennas (TAs) are activated within a single symbol duration (SD), whereas extra implicit information bits can be conveyed via the activated TA indices. The further refinements of SM or GSM techniques include differential SM [20], quadrature SM $[21,22]$ and rectangular differential SM [23] etc., which tend to focus on either improving the bit error rate (BER) performance or the throughput of SM. Additionally, SM in combination with MIMO systems was considered in [24, 25], where Xiao et al. [25] proposed spatial multiplexing aided SM, which activates multiple TAs. As a further development, Basar et al. [24] investigated space-time block coded SM (STBC-SM), combining their advantages.

MIMO or SM-aided multiple access systems have been widely investigated in [26-35]. In particular, the STBC-SCMA scheme of [31] achieved spatial-domain diversity, but suffered from both severe interference and high detection complexity when a high number of transmit antennas are employed. Fortunately, the SM-aided multiuser (MU) communication systems of $[26,27]$ successfully mitigated the interference, which reduced the number of RF chains thanks to the characteristics of SM, demonstrating superior BER performance over the family of conventional MU MIMO systems at the same spectral efficiency. Furthermore, the development of NOMA has also led to the design of SM-NOMA systems [28, 29,33, 35]. For instance, the SM-aided sparse code division multiple access (SM-SCDMA) system of [29] reduces the number of RF chains employed in MIMO-SCDMA systems, hence facilitating the implementation of message passing-aided (MPA) 
TABLE I

CONTRASTING THE CONTRIBUTIONS OF THIS WORK TO THE LITERATURE.

\begin{tabular}{l|c|c|c|c|c|c|c|c}
\hline Contributions & This work & [28] & [29] & [30] & [31] & [32] & [33] & [34] \\
\hline \hline Generalized spatial modulation (GSM) & $\checkmark$ & $\checkmark$ & & & & & & $\checkmark$ \\
\hline Space-time coding (STC) & $\checkmark$ & & & $\checkmark$ & $\checkmark$ & & & \\
\hline spatial- and frequency-domain (SFD) diversity & $\checkmark$ & & & & $\checkmark$ & & & $\checkmark$ \\
\hline Multicarrier (MC) communications & $\checkmark$ & & & & & & $\checkmark$ & $\checkmark$ \\
\hline Factor graph design & $\checkmark$ & & & & & $\checkmark$ & $\checkmark$ & \\
\hline Message passing-aided (MPA) detection & $\checkmark$ & & $\checkmark$ & $\checkmark$ & $\checkmark$ & & $\checkmark$ & $\checkmark$ \\
\hline Low-complexity detection & $\checkmark$ & $\checkmark$ & & & & $\checkmark$ & & \\
\hline BER over frequency-selective channels & $\checkmark$ & & $\checkmark$ & & & & $\checkmark$ & $\checkmark$ \\
\hline Union bound & $\checkmark$ & & $\checkmark$ & & $\checkmark$ & & $\checkmark$ & \\
\hline
\end{tabular}

detection. While the SM-SCDMA system of [33] exploits both spatial- and frequency-domain (SFD) resources, only FD diversity is achieved, since only a single RF chain is activated at a time, which motivates us to further explore the spatialdomain diversity for SM-aided NOMA systems. Recently, the sparse space-time-frequency spreading (SSTFS)-NOMA concept was proposed in [34], which attempts to exploit the multi-dimensional spreading at the transmitter side. However, the unique spreading code design required for SSTFS-NOMA system prevents its flexible application to more generalized SCMA system concepts or to MIMO systems having more than two TAs. Additionally, its excessive detection complexity restricts its implementation in practice.

Against this backdrop, we are motivated to exploit the uplink (UL) transmit diversity potential of SM-SCDMA systems in the SFD. To achieve this ambitious objective, we propose a space-time coded GSM for SCDMA (STC/GSMSCDMA) systems along with a pair of detection algorithms. The properties of typical SM- or MIMO-NOMA systems [2834] found in the open literature is summarized in Table I. The main contributions of this paper are summarized as follows.

- Firstly, we propose a STC/GSM-SCDMA system for supporting the heavily-loaded multiuser (MU) multicarrier (MC) UL, while achieving a beneficial transmit diversity gain both in the spatial-domain and in the FD. In our STC/GSM-SCDMA system, GSM is employed both for reducing the number of RF chains and for transmitting extra information bits via the activated TA indices, while STC is employed for exploiting spatial-domain diversity. Meanwhile, by adopting the LDS concept in the FD, MC signalling can also be activated for mitigating the deleterious effects of frequency-selective fading. Furthermore, in contrast to the conventional orthogonal MU systems, where 'only' up to $100 \%$ normalized user-load can be attained, each orthogonal FD resource unit in the proposed STC/GSM-SCDMA system is capable of supporting more than 'just' a single user. Hence, our solution supports a high normalized user-load.

- Secondly, we design a joint factor graph for representing the connections of the proposed STC/GSM-SCDMA system, which combines the GSM symbols with the quadrature amplitude modulation (QAM) symbols and aggregates all observations at all RAs of each subcarrier. Then bespoke MPA detection is conceived based on the proposed joint factor graph.

- Thirdly, a 3-dimensional (3D) factor graph is designed, where the connections between users and TAs as well as those between subcarriers and RAs are separately illustrated, based on which a low-complexity tailor-made approximate message passing (AMP) detector is proposed. The AMP detector conceived in this paper imposes 1000 times lower computational complexity than the MPA detector, at the cost of a modest $2 \mathrm{~dB}$ signal-tonoise ratio (SNR) increase.

- Finally, the theoretical single-user performance bound is derived for the proposed STC/GSM-SCDMA system for transmission over frequency-selective Rayleigh fading channels, which is employed as the benchmark of the multiuser STC/GSM-SCDMA systems. Our simulation results also demonstrate that our STC/GSM-SCDMA system outperforms the MIMO-NOMA systems of [31, 33].

The rest of this paper is structured as follows. Section II describes the transmitter and receiver of the proposed STC/GSM-SCDMA system. A pair of detection algorithms is proposed in Section III, whereas the analysis of a single-user STC/GSM-SCDMA system is discussed in Section IV. Section $\mathrm{V}$ characterizes the numerical results of the STC/GSMSCDMA system's performance in different scenarios. Finally, our main conclusions are provided in Section VI.

\section{System ModeL}

In this section, we introduce the transmitter and receiver models of the proposed UL STC/GSM-SCDMA system in Sections II-A and II-B, respectively, along with the main assumptions used in the study.

\section{A. Transmitter Model}

Explicitly, we consider the UL of a multiuser MC communication system, where $K$ users simultaneously transmit their signals mapped to $N$ subcarriers and $N_{T}$ activated transmit antennas (TAs) per user to a single base station (BS) equipped with $N_{R}$ receiver antennas (RAs). Frequencyselective Rayleigh fading channels having $L$ CIR taps are considered. The STC/GSM-SCDMA transmitter of user $k(k \in$ $[1, K])$ is shown in Fig. 1 , where $N_{A}$ TAs out of the total $N_{T}$ TAs are activated to transmit user $k$ 's signal. 


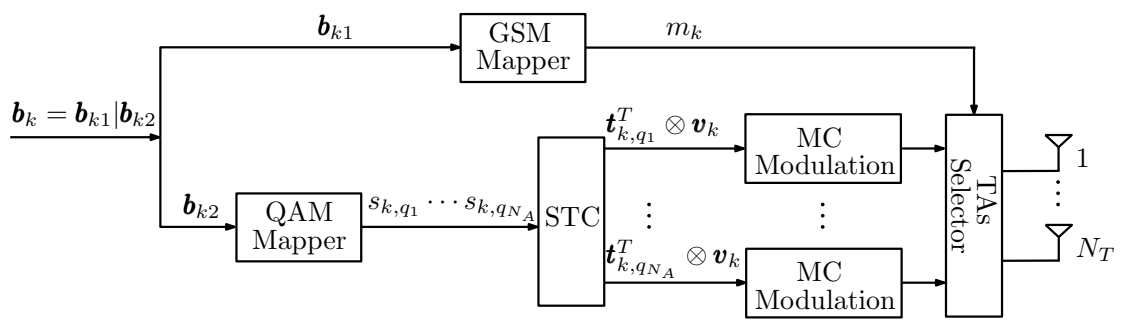

Fig. 1. The transmitter schematic diagram of the $k$ th user in the STC/GSM-SCDMA system.

TABLE II

THE BIT-GSM SYMBOL-TA MAPPING FOR A $N_{T}=6, N_{A}=2$ STC/GSM-SCDMA SYSTEM.

\begin{tabular}{c|c|c}
\hline $\boldsymbol{b}_{k 1}$ & $\mathcal{M}_{1}$ & TA indices \\
\hline \hline 000 & $\bar{m}_{1}$ & $(1,4)$ \\
\hline 001 & $\bar{m}_{2}$ & $(1,5)$ \\
\hline 010 & $\bar{m}_{3}$ & $(1,6)$ \\
\hline 011 & $\bar{m}_{4}$ & $(2,4)$ \\
\hline 100 & $\bar{m}_{5}$ & $(2,5)$ \\
\hline 101 & $\bar{m}_{6}$ & $(2,6)$ \\
\hline 110 & $\bar{m}_{7}$ & $(3,5)$ \\
\hline 111 & $\bar{m}_{8}$ & $(3,6)$ \\
\hline
\end{tabular}

More specifically, within a single symbol duration (SD), user $k$ transmits the bit sequence $\boldsymbol{b}_{k}=\boldsymbol{b}_{k 1} \mid \boldsymbol{b}_{k 2}$ of $b=b_{1}+b_{2}$ bits by $N_{A}$ activated TAs chosen from the $N_{T}$ TAs. Here, $\boldsymbol{b}_{k 1}$ comprising $b_{1}=\left\lfloor\log _{2}\left(\begin{array}{c}N_{T} \\ N_{A}\end{array}\right)\right\rfloor^{1}$ bits is employed to activate $N_{A}$ TA indices, forming a GSM symbol $m_{k}$ in the GSM symbol set $\mathcal{M}_{1}=\left\{\bar{m}_{1}, \cdots, \bar{m}_{M_{1}}\right\}$, where $M_{1}=2^{b_{1}}$. Table II exemplifies the bit-GSM symbol-TA mapping for a $N_{T}=6, N_{A}=2 \mathrm{STC} / \mathrm{GSM}$-SCDMA system. Hence, there exist $\left(\begin{array}{l}N_{T} \\ N_{A}\end{array}\right)=\left(\begin{array}{l}6 \\ 2\end{array}\right)=15$ activated TA combinations, which indicates that $b_{1}=\left\lfloor\log _{2} 15\right\rfloor=3$ bits can be conveyed by the TA activation patterns [19]. For instance, as shown in Table II, $\boldsymbol{b}_{k 1}=[000]$ maps to the GSM symbol $\bar{m}_{1}$, which activates the $q_{1}=1$-st and $q_{2}=4$-th TAs to transmit user $k$ 's signal. Meanwhile, a $\left(b_{2}=N_{A} \log _{2} M_{2}\right)$-bit sequence $\boldsymbol{b}_{k 2}$ is mapped to $N_{A}$ $M_{2}$ QAM symbols, $\underbrace{s_{k, q_{1}}, s_{k, q_{2}}, \cdots, s_{k, q_{N_{A}}}}_{N_{A}}$, where $s_{k, q_{a}} \in \mathcal{S}$,

$\mathcal{S}$ is the QAM symbol set and $q_{a}, a=1, \cdots, N_{A}$, is the antenna index of the $a$-th activated TA.

Later, STC is performed on the $N_{A}$ symbols $\underbrace{s_{k, q_{1}}, s_{k, q_{2}}, \cdots, s_{k, q_{N_{A}}}}_{N_{A}}$ of user $k$ to achieve transmit diversity in the spatial domain. The well-known Alamouti STC code is formulated as [36]

$$
\mathcal{G}_{k, 2}=\frac{1}{\sqrt{2}}\left[\begin{array}{cc}
s_{k, q_{1}} & -s_{k, q_{2}}^{*} \\
s_{k, q_{2}} & s_{k, q_{1}}^{*}
\end{array}\right],
$$

where $s_{k, q_{1}}$ and $s_{k, q_{2}}$ represent the QAM symbols transmitted by the $q_{1}$-th and $q_{2}$-th TAs, respectively, in the $z=1$-st SD, $-s_{k, q_{2}}^{*}$ and $s_{k, q_{1}}^{*}$ denote the QAM symbols transmitted by the $q_{1}$-th and $q_{2}$-th TA, respectively, in the $z=2$-nd SD.

In a more general form, the STC-coded QAM symbol of a general STC/GSM-SCDMA system employing $N_{A}$ TA over

\footnotetext{
${ }^{1}\lfloor x\rfloor$ represents the largest integer that is smaller than $x$.
}

the $Z$ SDs can be expressed as

$$
\boldsymbol{T}_{k}=\mathcal{G}_{N_{A}, k}^{(Z)}=\frac{1}{\sqrt{Z}}\left[\begin{array}{cccc}
t_{k, q_{1}}^{(1)} & t_{k, q_{1}}^{(2)} & \cdots & t_{k, q_{1}}^{(Z)} \\
t_{k, q_{2}}^{(1)} & t_{k, q_{2}}^{(2)} & \cdots & t_{k, q_{2}}^{(Z)} \\
\vdots & \vdots & \ddots & \vdots \\
t_{k, q_{N_{A}}}^{(1)} & t_{k, q_{N_{A}}}^{(2)} & \cdots & t_{k, q_{N_{A}}}^{(Z)}
\end{array}\right]
$$

where $\mathcal{G}_{N_{A}, k}^{(Z)}$ represents the $\left(N_{A} \times Z\right)$-dimensional STC structure and $t_{k, q_{a}}^{(z)}, a=1, \cdots, N_{A}$, and $z=1, \cdots, Z$, is the STCcoded QAM symbol transmitted by $q_{a}$-th TA in the $z$-th SD. Since the QAM symbol $s_{k, q_{a}}$ will be transmitted after STC in its original form $s_{k, q_{a}}$, or as $s_{k, q_{a}}^{*}$, or as $-s_{k, q_{a}}$, or as $-s_{k, q_{a}}^{*}$ in the $z$-th SD, we define $\tilde{\mathcal{S}}^{(z)}$ as the 'STC-transformed' QAM symbol set in the $z$-th SD. Again, considering the Alamouti-coded QAM symbols of (1) transmitted by the $q_{1}$ th TA as an example, we have $t_{k, q_{1}}^{(1)}=s_{k, q_{1}}^{(1)} \in \tilde{\mathcal{S}}^{(1)}=\mathcal{S}$, which indicates that in the 1 -st $\mathrm{SD}, s_{k, q_{1}}^{(1)}$ is selected from the original set $\mathcal{S}$ without any transformation. By contrast, we have $t_{k, q_{1}}^{(2)}=-s_{k, q_{2}}^{*(2)} \in \tilde{\mathcal{S}}^{(2)}=-\mathcal{S}^{*}$, which means that in the 2-nd SD, the QAM symbol conveyed by the $q_{1}$-th TA will be transmitted in the form of $t_{k, q_{1}}^{(2)}=-s_{k, q_{2}}^{*(2)}$, where each symbol in the QAM symbol set $\mathcal{S}$ should be in this specific form, giving $\tilde{\mathcal{S}}^{(2)}=-\mathcal{S}^{*}$.

Following the STC, each STC-coded QAM symbol $t_{k, q_{a}}^{(z)}$ is sparsely spread over $d_{c}$ subcarriers by adopting the $(N \times 1)$ LDS $\boldsymbol{v}_{k}$ preassigned to user $k$. The $d_{c}$ non-zero elements in the sparse sequence $\boldsymbol{v}_{k}$ select the subcarriers employed for transmitting the QAM symbols of user $k$ and are normalized to satisfy $\left\|\boldsymbol{v}_{k}\right\|^{2}=1$. The positions of the non-zero elements in $\boldsymbol{v}_{k}$ can be represented by an indicator matrix $\boldsymbol{V}=\left[\overline{\boldsymbol{v}}_{1}, \overline{\boldsymbol{v}}_{2}, \cdots, \overline{\boldsymbol{v}}_{K}\right]$, where $\overline{\boldsymbol{v}}_{k}$ is comprised of '1's and '0's representing the positions of non-zero elements and those of zero elements, respectively, in $\boldsymbol{v}_{k}$. For instance, for a $N=4$, $K=6 \mathrm{STC} / \mathrm{GSM}$-SCDMA system, we may have an indicator matrix $V$ expressed as

$$
\boldsymbol{V}=\left[\begin{array}{llllll}
1 & 1 & 1 & 0 & 0 & 0 \\
1 & 0 & 0 & 1 & 1 & 0 \\
0 & 1 & 0 & 1 & 0 & 1 \\
0 & 0 & 1 & 0 & 1 & 1
\end{array}\right]
$$

Correspondingly, the number of users sharing one of the $N$ subcarriers is expressed as $d_{x}$, which has to obey $d_{x} \ll K$. Then, in the example of the Alamouti's code, the $(2 N \times 2)$ - 
dimensional transmit signal matrix can be formulated as

$$
\boldsymbol{T}_{k} \otimes \boldsymbol{v}_{k}=\left[\begin{array}{cc}
s_{k, q_{1}} & -s_{k, q_{2}}^{*} \\
s_{k, q_{2}} & s_{k, q_{1}}^{*}
\end{array}\right] \otimes \boldsymbol{v}_{k}=\left[\begin{array}{cc}
t_{k, q_{1}}^{(1)} & t_{k, q_{1}}^{(2)} \\
t_{k, q_{2}}^{(1)} & t_{k, q_{2}}^{(2)}
\end{array}\right] \otimes \boldsymbol{v}_{k},
$$

where $\otimes$ is the Kronecker product.

\section{B. Receiver Model}

Let us express the $L$-path channel impulse response (CIR) $\boldsymbol{h}_{k, p}^{(r)}$ between the $p$-th TA $\left(p=1,2, \cdots, N_{T}\right)$ of user $k$ and the $r$-th RA $\left(r=1,2, \cdots, N_{R}\right)$ at the BS in the TD as

$$
\boldsymbol{h}_{k, p}^{(r)}=\left[h_{k, p, 1}^{(r)}, h_{k, p, 2}^{(r)}, \cdots, h_{k, p, L}^{(r)}\right]^{T},
$$

where $\boldsymbol{h}_{k, p}^{(r)}$ are independent identically distributed (iid) complex Gaussian random vectors with a zero mean and a variance of $0.5 / L$ per dimension. Then, the corresponding FD channel transfer function (FD-CHTF) $\overline{\boldsymbol{h}}_{k, p}^{(r)}$ experienced by the $N$ subcarriers can be expressed as [37]

$$
\overline{\boldsymbol{h}}_{k, p}^{(r)}=\mathcal{F} \boldsymbol{\Phi}_{L} \boldsymbol{h}_{k, p}^{(r)},
$$

where $\boldsymbol{\Phi}_{L}$ is an $(N \times L)$ mapping matrix constituted by the first $L$ columns of an identity matrix $\boldsymbol{I}_{N}$, and $\mathcal{F}$ is the $(N \times N)$ FFT matrix having the property of $\mathcal{F F}^{H}=\mathcal{F}^{H} \mathcal{F}=N I_{N}$. When considering all the $\left(N_{T} \times N_{R}\right)$ antennas, the $\left(N N_{R} \times\right.$ $N_{T}$ )-dimensional FD channel matrix $\overline{\boldsymbol{H}}_{k}$ of user $k$ can be collectively expressed as

$$
\overline{\boldsymbol{H}}_{k}=\left[\begin{array}{ccc}
\overline{\boldsymbol{h}}_{k, 1}^{(1)} & \cdots & \overline{\boldsymbol{h}}_{k, N_{T}}^{(1)} \\
\vdots & \ddots & \vdots \\
\overline{\boldsymbol{h}}_{k, 1}^{\left(N_{R}\right)} & \cdots & \overline{\boldsymbol{h}}_{k, N_{T}}^{\left(N_{R}\right)}
\end{array}\right]
$$

Similar to the assumption employed in STC, we assume that the CIR $\overline{\boldsymbol{h}}_{k, p}^{(r)}$ of the proposed STC/GSM-SCDMA system remains constant across the $Z$ SDs of one STC frame. Therefore, the $(N \times Z)$-dimensional received observations $\boldsymbol{Y}^{(r)}$ at the $r$-th RA over the $Z$ SDs of a STC/GSM-SCDMA system having $N_{A}$ activated TAs can be expressed as

$$
\begin{aligned}
\boldsymbol{Y}^{(r)}= & \frac{1}{\sqrt{Z}} \sum_{k=1}^{K}\left[\operatorname{diag}\left(\overline{\boldsymbol{h}}_{k, q_{1}}^{(r)}\right), \operatorname{diag}\left(\overline{\boldsymbol{h}}_{k, q_{2}}^{(r)}\right), \cdots, \operatorname{diag}\left(\overline{\boldsymbol{h}}_{k, q_{N_{A}}}^{(r)}\right)\right] \\
& \times\left(\boldsymbol{T}_{k} \otimes \boldsymbol{v}_{k}\right)+\boldsymbol{N}^{(r)} \\
= & \frac{1}{\sqrt{Z}} \sum_{k=1}^{K} \operatorname{diag}\left(\boldsymbol{v}_{k}\right)\left[\overline{\boldsymbol{h}}_{k, q_{1}}^{(r)} \ldots, \overline{\boldsymbol{h}}_{k, q_{N_{A}}}^{(r)}\right] \boldsymbol{T}_{k}+\boldsymbol{N}^{(r)} \\
= & \frac{1}{\sqrt{Z}} \sum_{k=1}^{K} \operatorname{diag}\left(\boldsymbol{v}_{k}\right) \overline{\boldsymbol{H}}_{m_{k}}^{(r)} \boldsymbol{T}_{k}+\boldsymbol{N}^{(r)}
\end{aligned}
$$

where $\operatorname{diag}(\cdot)$ represents the diagonal matrix constructed by a vector, $\overline{\boldsymbol{H}}_{m_{k}}^{(r)}=\left[\overline{\boldsymbol{h}}_{k, q_{1}}^{(r)} \ldots, \overline{\boldsymbol{h}}_{k, q_{N_{A}}}^{(r)}\right]$ is a $\left(N \times N_{A}\right)$ dimensional channel matrix representing the FD CIRs between the $N_{A}$ activated TAs selected by $m_{k} \in \mathcal{M}_{1}$ and the $r$ th RA at the BS. Additionally, $\boldsymbol{N}^{(r)}$ is the additive white Gaussian noise (AWGN) with each column obeying the Gaussian distribution with zero-mean and a covariance matrix of
$2 \sigma^{2} \boldsymbol{I}_{N}$, which may be expressed as $\mathcal{C N}\left(0,2 \sigma^{2} \boldsymbol{I}_{N}\right)$, where $\sigma^{2}=1 /(2 \gamma), \gamma$ denotes the SNR per symbol.

If we define the received observations $Y$ of $N_{r}$ RAs over the $Z$ SDs of a STC/GSM-SCDMA system having $N_{A}$ activated TAs as $\boldsymbol{Y}=\left[\left(\boldsymbol{Y}^{(1)}\right)^{T},\left(\boldsymbol{Y}^{(2)}\right)^{T}, \cdots,\left(\boldsymbol{Y}^{\left(N_{R}\right)}\right)^{T}\right]^{T}$ and let $\overline{\boldsymbol{H}}_{m_{k}}=\left[\left(\overline{\boldsymbol{H}}_{m_{k}}^{(1)}\right)^{T},\left(\overline{\boldsymbol{H}}_{m_{k}}^{(2)}\right)^{T}, \cdots,\left(\overline{\boldsymbol{H}}_{m_{k}}^{\left(N_{R}\right)}\right)^{T}\right]^{T}$, then we have

$$
\begin{aligned}
\boldsymbol{Y} & =\frac{1}{\sqrt{Z}} \sum_{k=1}^{K}\left(\boldsymbol{I}_{N_{R}} \otimes \operatorname{diag}\left(\boldsymbol{v}_{k}\right)\right) \overline{\boldsymbol{H}}_{m_{k}} \boldsymbol{T}_{k}+\boldsymbol{n} \\
& =\frac{1}{\sqrt{Z}} \sum_{k=1}^{K}\left(\boldsymbol{I}_{N_{R}} \otimes \operatorname{diag}\left(\boldsymbol{v}_{k}\right)\right) \overline{\boldsymbol{H}}_{k} \boldsymbol{E}_{m_{k}} \boldsymbol{T}_{k}+\boldsymbol{n} \\
& =\frac{1}{\sqrt{Z}} \sum_{k=1}^{K} \boldsymbol{H}_{k} \boldsymbol{E}_{m_{k}} \boldsymbol{T}_{k}+\boldsymbol{n},
\end{aligned}
$$

where by definition, we have $\boldsymbol{H}_{k}=\left(\boldsymbol{I}_{N_{R}} \otimes \operatorname{diag}\left(\boldsymbol{v}_{k}\right)\right) \overline{\boldsymbol{H}}_{k}$, with $\overline{\boldsymbol{H}}_{k}$ given by (7) and $\boldsymbol{E}_{m_{k}}$ is a $\left(N_{T} \times N_{A}\right)$ dimensional TA selection matrix, whose $a$-th column has only the $q_{a}$-th ( $a=1, \cdots, N_{A}$ ) element set to ' 1 ', while all the remaining elements are ' 0 '.

Having obtained the MIMO observation equation of (9), let us now consider both our new MPA and AMP based detector in the next section.

\section{Detection Algorithms}

In Sections III-A and III-B, we propose the above pair of detectors for our STC/GSM-SCDMA system, respectively, following the introduction of two different factor graph representations of the received signals.

\section{A. Message Passing-Aided Detection}

Similar to other code-domain NOMA systems, the usersubcarrier relationship of the STC/GSM-SCDMA system can be visualized by a joint factor graph, comprising $K$ variable nodes (VNs), representing the $K$ symbols transmitted by $K$ users, and $N$ check nodes (CNs), related to the observations on $N$ subcarriers. In contrast to the conventional factor graph, where a single $\mathrm{VN}$ is only associated with a single QAM symbol, the $k$-th $(k \in[1, K]) \mathrm{VN}$ in our proposed joint factor graph combines not only the GSM symbol determined by the activated TAs, but also the $N_{A}$ QAM symbols transmitted by $N_{A}$ activated TAs of user $k$, whereas, the $n$-th $(n \in[1, N])$ $\mathrm{CN}$ is comprised of the $N_{R}$ observations from $N_{R}$ RAs of the $n$-th subcarrier. For convenience, let us define the GSM-QAM symbol transmitted by the $N_{A}$ activated TAs of user $k$ as $u_{k}=$ $\left(m_{k} \mid s_{k, q_{1}}, s_{k, q_{2}}, \cdots, s_{k, q_{N_{A}}}\right)$, with $u_{k} \in \mathcal{M}=\mathcal{M}_{1} \otimes \mathcal{S}^{N_{A}}$.

The joint factor graph of our the STC/GSM-SCDMA system having $N=4$ subcarriers, $d_{c}=2$ and $d_{x}=3$, supporting $K=6$ users is exemplified in Fig. 2.

Before we detail our MPA detector, let us first define the connections to the $\mathrm{VN} k$ and those to the $\mathrm{CN} n$ in the factor graph into two sets, which can be respectively expressed as

$$
\begin{array}{ll}
\mathcal{U}_{k}=\left\{n: 1 \leqslant n \leqslant N, e_{k, n} \neq 0\right\}, \quad k=1, \cdots, K, \\
\mathcal{C}_{n}=\left\{k: 1 \leqslant k \leqslant K, e_{k, n} \neq 0,\right\}, \quad n=1, \cdots, N .
\end{array}
$$




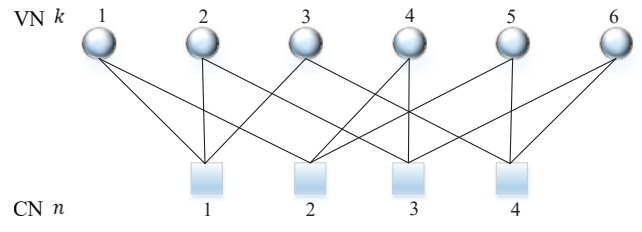

Fig. 2. An example of the proposed joint factor graph design of the STC/GSM-SCDMA system with the parameters of $N=4, d_{c}=2, d_{x}=3$ and $K=6$.

As shown in Fig. 2, information can be conveyed both upwards from the $\mathrm{CN} n$ to $\mathrm{VN} k$ and downward from $\mathrm{VN} k$ to $\mathrm{CN} n$. Let us define $\delta_{n \rightarrow k}^{(g, i)}(g \in \mathcal{M})$ as the information conveyed from $\mathrm{CN} n$ to $\mathrm{VN} k$ during the $i$-th iteration of the MPA detection, which represents the probability of $g=u_{k}$, when given the probabilities received by $\mathrm{CN} n$ from all the connected VNs $[n]$, excluding VN $k$, which can be expressed as $[n] \backslash k \in\left\{\mathcal{C}_{n} \backslash k\right\}$, where ' $\backslash$ ' represents the operation of exclusion. By contrast, the information transmitted from VN $k$ to $\mathrm{CN} n$ in the $i$-th iteration is expressed as $\eta_{k \rightarrow n}^{(g, i)}$, which represents the probability of $g=u_{k}$, given the probabilities received by $\mathrm{VN} k$ from all the connected $\mathrm{CNs}[k]$ excluding CN $n$, which can be expressed as $[k] \backslash n \in\left\{\mathcal{U}_{k} \backslash n\right\}$. Then the MPA based detector [33] operates as follows:

1) Initialisation: Since we assume equiprobable transmission of all symbols, $\eta_{k \rightarrow n}^{(g, 0)}$ is initialised to $1 / M$ for all $g=u_{k} \in \mathcal{M}$ and any $e_{k \rightarrow n} \neq 0$, where $M=M_{1} \times M_{2}^{N_{A}}$.

2) VN update: Then, at the $i$-th iteration, the information conveyed by $\mathrm{CN} n$ to $\mathrm{VN} k$, i.e. $\delta_{n \rightarrow k}^{(g, i)}$ for $k \in \mathcal{C}_{n}$ and $n \in \mathcal{U}_{k}$ is the product of the information gleaned from all the other edges connected to $\mathrm{CN} n$, whereas the information received at $\mathrm{VN} k$ is the sum of the information arriving from all the CNs that are connected to $\mathrm{VN} k$, which can be expressed as

$$
\begin{aligned}
\delta_{n \rightarrow k}^{g, i}= & \sum_{\boldsymbol{u}_{[n]} \in \mathcal{M}^{d_{x}-1}}\left(\prod_{u_{k^{\prime}} \in \boldsymbol{u}_{[n]} \backslash u_{k}} \eta_{k^{\prime} \rightarrow n}^{(g, i)}\right) \\
& \times \prod_{r=1}^{N_{R}} \prod_{z=1}^{Z} p\left(y_{n r}^{(z)} \mid \boldsymbol{u}_{[n]}, u_{k}=g\right),
\end{aligned}
$$

where $\mathcal{M}^{d_{x}-1}$ represents the GSM-QAM symbol sets of the $\left(d_{x}-1\right)$ users connected to $\mathrm{CN} n$ excluding user $k$ and $\prod_{u_{k^{\prime}} \in \boldsymbol{u}_{[n]} \backslash u_{k}} \eta_{k^{\prime} \rightarrow n}^{(g, i)}$ is the a priori probability of a given $\boldsymbol{u}_{[n]} \backslash u_{k}$ with $u_{k^{\prime}}=g$. When $\boldsymbol{u}_{[n]}$ is given, the PDF of $p\left(y_{n r}^{(z)} \mid \boldsymbol{u}_{[n]}\right)$ can be expressed as

$$
\begin{aligned}
& p\left(y_{n r}^{(z)} \mid \boldsymbol{u}_{[n]}\right)=\frac{1}{2 \pi \sigma^{2}} \\
& \times \exp \left(-\frac{\left|y_{n r}^{(z)}-\frac{1}{\sqrt{Z}} \sum_{k \in \mathcal{C}_{n}} \sum_{q_{a} \in m_{k}} h_{k, q_{a}, n}^{(r)} t_{k, q_{a}}^{(z)}\right|^{2}}{2 \sigma^{2}}\right),
\end{aligned}
$$

where $q_{a} \in m_{k}$ represents the activated TA selected by the GSM symbol $m_{k}$ and $\mathcal{C}_{n}$ is given in (11).

In particular, when Alamouti's code is employed, we have

$$
\begin{aligned}
& N_{A}=2, Z=2 \text {, and } \\
& p\left(y_{n r}^{(1)} \mid \boldsymbol{u}_{[n]}\right)=\frac{1}{2 \pi \sigma^{2}} \\
& \times \exp \left(-\frac{\left|y_{n r}^{(1)}-\frac{1}{\sqrt{2}} \sum_{k \in \mathcal{C}_{n}}\left(h_{k, q_{1}, n}^{(r)} s_{k, q_{1}}+h_{k, q_{2}, n}^{(r)} s_{k, q_{2}}\right)\right|^{2}}{2 \sigma^{2}}\right),
\end{aligned}
$$

$$
\begin{aligned}
& p\left(y_{n r}^{(2)} \mid \boldsymbol{u}_{[n]}\right)=\frac{1}{2 \pi \sigma^{2}} \\
& \times \exp \left(-\frac{\left|y_{n r}^{(2)}-\frac{1}{\sqrt{2}} \sum_{k \in \mathcal{C}_{n}}\left(-h_{k, q_{1}, n}^{(r)} s_{k, q_{2}}^{*}+h_{k, q_{2}, n}^{(r)} s_{k, q_{1}}^{*}\right)\right|^{2}}{2 \sigma^{2}}\right) .
\end{aligned}
$$

3) $C N$ update: Next, at the $(i+1)$-st iteration, the values $\delta_{n \rightarrow k}^{(g, i)}$ obtained in the $i$-th iteration are used to update $\eta_{k \rightarrow n}^{(g, i+1)}$ which can be expressed as

$$
\eta_{k \rightarrow n}^{(g, i+1)}=\varepsilon_{k, n} \prod_{n^{\prime} \in \mathcal{U}_{k} \backslash n} \delta_{n^{\prime} \rightarrow k}^{(g, i)},
$$

where $\varepsilon_{k, n}$ is the normalisation factor enabling $\sum_{g \in \mathcal{M}} \eta_{k \rightarrow n}^{(g, i+1)}=1$.

4) Symbol mapping: Finally, after the pre-set number of iterations $I$ is reached, the detector detects the transmitted symbol of the $k$ th user as

$$
\begin{aligned}
\hat{u}_{k} & =\left(\hat{m}_{k} \mid \hat{s}_{k, q_{1}}, \cdots, \hat{s}_{k, q_{N_{A}}}\right) \\
& =\arg \max _{g \in \mathcal{M}} \prod_{n \in \mathcal{U}_{k}} \delta_{n \rightarrow k}^{(g, I)} .
\end{aligned}
$$

We can see from (12) that the computational complexity is primarily dominated by the upward information transition, giving a complexity order of $\mathcal{O}\left(M^{d_{x}-1}\right)=\mathcal{O}\left(\left(M_{1} M_{2}^{N_{A}}\right)^{d_{x}-1}\right)$.

\section{B. Approximated Message Passing Detection}

From the above discussion we know that the MPA complexity order is $\mathcal{O}\left[\left(M_{1} M_{2}^{N_{A}}\right)^{d_{x}-1}\right]$, which increases exponentially with the number of users $d_{x}$ sharing a single subcarrier, and the number of TAs activated per symbol. In order to reduce the detection complexity, we now propose a novel detection algorithm for our STC/GSM-SCDMA system by appropriately adapting the AMP algorithm [38,39], which approximates the downward propagation of STC-coded QAM symbol $t_{k, q_{a}}^{(z)}$ transmitted by $q_{a}$-th TA of user $k$ at $z$-th SD to obey a complex Gaussian distribution $\mathcal{N}_{\mathbb{C}}\left(t_{k, q_{a}}^{(z)} ; \mu_{k m_{k} \rightarrow n r}^{\left(q_{a}, z, i\right)}, \omega_{k m_{k} \rightarrow n r}^{\left(q_{a}, z, i\right)}\right)[39,40]$, where $\mu_{k m_{k} \rightarrow n r}^{\left(q_{a}, z, i\right)}$ and $\omega_{k m_{k} \rightarrow n r}^{\left(q_{a}, z, i\right)}$ represent the mean and variance of the complex random variable $t_{k, q_{a}}^{(z)}$, respectively.

Now that the STC-coded QAM symbols and the GSM symbol are detected separately, we design a 3D factor graph representation of the STC/GSM-SCDMA systems, as exemplified in Fig. 3, before we introduce the AMP detection. More specifically, VN $k$ in the joint factor graph of Fig. 2 is now expanded to $M_{1}$ sub-nodes, which represent the $M_{1}$ GSM symbols that are possibly employed to select user $k$ 's activated TAs. Hence, VN $k m_{k}$ represents the $N_{A}$ activated TAs of user 
$k$ determined by the GSM symbol $m_{k} \in \mathcal{M}_{1}$, as exemplified in Table II. By contrast, each $\mathrm{CN}$ in the 3D factor graph now combines all the $N_{R}$ observations obtained from the $N_{R}$ RAs, such that CN $n r\left(n=1,2, \cdots, N, r=1,2, \cdots, N_{R},\right)$ represents the observation of the $n$-th subcarrier at the $r$-th $\mathrm{RA}$ at the receiver.

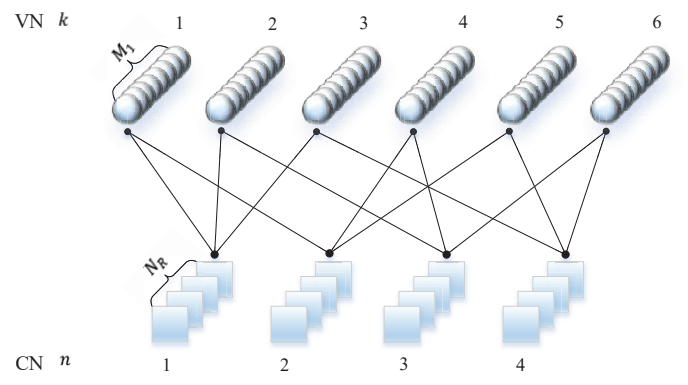

Fig. 3. An example of the proposed 3D factor graph design of the STC/GSMSCDMA system with the parameters of $N=4, d_{c}=2, d_{x}=3$ and $K=6$.

Therefore, the connections to $\mathrm{VN} k m_{k}$ and to $\mathrm{CN} n r$ in the proposed 3D factor graph can then be defined in two sets, expressed as

$$
\begin{gathered}
\mathcal{U}_{k m_{k}}=\left\{n: 1 \leqslant n \leqslant N, r: 1 \leqslant r \leqslant N_{R}, e_{k m_{k}, n r} \neq 0\right\} \\
k=1, \cdots, K, m_{k} \in \mathcal{M}_{1} \\
\mathcal{C}_{n r}=\left\{m_{k} \in \mathcal{M}_{1}, k: 1 \leqslant k \leqslant K, e_{k m_{k}, n r} \neq 0\right\} \\
n=1, \cdots, N, r=1, \cdots, N_{R}
\end{gathered}
$$

Similar to the MPA detection, the AMP detection also passes information both upwards and downwards via the 3D factor graph of Fig. 3. Now let us define the information associated with $t_{k, q_{a}}^{(z)}$ passed from $\mathrm{VN} k m_{k}$ to $\mathrm{CN} n r$ in the $i$-th iteration as $\eta_{k m_{k} \rightarrow n r}^{(i)}\left(t_{k, q_{a}}^{(z)}\right)$, whereas the reverse information transmission from $\mathrm{CN} n r$ to $\mathrm{VN} k m_{k}$ in the $i$-th iteration as $\delta_{n r \rightarrow k m_{k}}^{(i)}\left(t_{k, q_{\alpha}}^{(z)}\right)$. Then, the proposed AMP detector for the STC/GSM-SCDMA system operates the following steps:

1) Initialisation: The initialisation of the information $\eta_{k m_{k} \rightarrow n r}^{(0)}\left(t_{k, q_{a}}^{(z)}\right)$ from VN $k m_{k}$ to CN $n r$ is under the assumption of equiprobable transmission, which can be expressed as $\eta_{k m_{k} \rightarrow n r}^{(0)}\left(t_{k, q_{a}}^{(z)}\right)=1 / M$. Hence, in the 1 -st iteration we have the mean $\mu_{k m_{k} \rightarrow n r}^{\left(q_{a}, z, 1\right)}$ and variance $\omega_{k m_{k} \rightarrow n r}^{\left(q_{a}, z, 1\right)}$ for the Gaussian distributed variable $t_{k, q_{a}}^{(z)}$, which are expressed as

$$
\begin{aligned}
\mu_{k m_{k} \rightarrow n r}^{\left(q_{a}, z, 1\right)}= & \sum_{t_{k, q_{a}}^{(z)} \in \tilde{\mathcal{S}}^{(z)}} t_{k, q_{a}}^{(z)} \cdot \eta_{k m_{k} \rightarrow n r}^{(0)}\left(t_{k, q_{a}}^{(z)}\right) \\
= & \frac{1}{M} \sum_{t_{k, q_{a}}^{(z)} \in \tilde{\mathcal{S}}^{(z)}} t_{k, q_{a}}^{(z)} \\
\omega_{k m_{k} \rightarrow n r}^{\left(q_{a}, z, 1\right)}= & \sum_{t_{k, q_{a}}^{(z)} \in \tilde{\mathcal{S}}^{(z)}}\left|t_{k, q_{a}}^{(z)}\right|^{2} \cdot \eta_{k m_{k} \rightarrow n r}^{(0)}\left(t_{k, q_{a}}^{(z)}\right) \\
& -\left|\mu_{k m_{k} \rightarrow n r}^{\left(q_{a}, z, 1\right)}\right|^{2},
\end{aligned}
$$

respectively, where $\tilde{\mathcal{S}}^{(z)}$ represents the 'STC-transformed' QAM symbol set of user $k$ in the $z$-th $\mathrm{SD}$, as defined in Section II-A.

2) VN update: Rather than updating $\eta_{k m_{k} \rightarrow n r}^{(i)}\left(t_{k, q_{a}}^{(z)}\right)$ using (12), as that in MPA detector, the AMP detector approximates $\eta_{k m_{k} \rightarrow n r}^{(i)}\left(t_{k, q_{a}}^{(z)}\right)$ as a complex Gaussian variable with the distribution $\mathcal{N}_{\mathbb{C}}\left(t_{k, q_{a}}^{(z)} ; \mu_{k m_{k} \rightarrow n r}^{\left(q_{a}, z, i\right)}, \omega_{k m_{k} \rightarrow n r}^{\left(q_{a}, z, i\right)}\right)$, which will be derived after the description of the $\mathrm{CN}$ update in the next step, as given in (29) and (30).

Then, in the $i$-th iteration of the AMP detection, the update of $\delta_{n r \rightarrow k m_{k}}^{(i)}\left(t_{k, q_{a}}^{(z)}\right)$ is similar to that of the MPA detector, which is also the product of the information obtained from all the other edges connected to $\mathrm{CN} n r$, and can be expressed as

$$
\begin{aligned}
& \delta_{n r \rightarrow k m_{k}}^{(i)}\left(t_{k, q_{a}}^{(z)}\right)=\sum_{\boldsymbol{u}_{[n] \backslash\left(m_{k} \mid t_{k, q_{a}}^{(z)}\right)}} \prod_{\left.\prod_{k^{\prime} m_{k}^{\prime} \in \mathcal{C}_{n r} \backslash k m_{k}} \prod_{q_{a}^{\prime} \in m_{k}^{\prime}} \mathcal{N}_{\mathbb{C}}\left(t_{k^{\prime}, q_{a}^{\prime}}^{(z)} ; \mu_{k^{\prime} m_{k}^{\prime} \rightarrow n r}^{\left(q_{a}^{\prime}, z, i\right)}, \omega_{k^{\prime} m_{k}^{\prime} \rightarrow n r}^{\left(q^{\prime}, z, i\right)}\right)\right)} \times p\left(y_{n r}^{(z)} \mid \boldsymbol{u}_{[n]}\right), \\
& =\mathcal{N}_{\mathbb{C}}\left(h_{k, q_{a}, n}^{(r)} t_{k, q_{a}}^{(z)} ; \alpha_{n r \rightarrow k m_{k}}^{\left(q_{a}, z, i\right)}, \beta_{n r \rightarrow k m_{k}}^{\left(q_{a}, z, i\right)}\right),
\end{aligned}
$$

where $p\left(y_{n r}^{(z)} \mid \boldsymbol{u}_{[n]}\right)$ is given by (13) and is also Gaussian distributed. Hence, $\delta_{n r \rightarrow k m_{k}}^{(i)}\left(t_{k, q_{a}}^{(z)}\right)$ can also be approximated by a Gaussian distribution $\mathcal{N}_{\mathbb{C}}\left(h_{k, q_{a}, n}^{(r)} t_{k, q_{a}}^{(z)} ; \alpha_{n r \rightarrow k m_{k}}^{\left(q_{a}, z, i\right)}, \beta_{n r \rightarrow k m_{k}}^{\left(q_{a}, z, i\right)}\right)$, as shown in (22b), where $\alpha_{n r \rightarrow k m_{k}}^{\left(q_{a}, z, i\right)}$ and $\beta_{n r \rightarrow k m_{k}}^{\left(q_{a}, z, i\right)}$ are the mean and variance of the variable $h_{k, q_{a}, n}^{(r)} t_{k, q_{a}}^{(z)}$ and can be expressed as

$$
\begin{aligned}
\alpha_{n r \rightarrow k m_{k}}^{\left(q_{a}, z, i\right)}= & y_{n r}^{(z)}-\sum_{k^{\prime} m_{k}^{\prime} \in \mathcal{C}_{n r}} \sum_{q_{a}^{\prime} \in m_{k}^{\prime}}\left(h_{k^{\prime}, q_{a}^{\prime}, n}^{(r)} \mu_{k^{\prime} m_{k}^{\prime} \rightarrow n r}^{\left(q_{a}^{\prime}, z, i\right)}\right) \\
& +h_{k, q_{a}, n}^{(r)} \mu_{k m_{k} \rightarrow n r}^{\left(q_{a}, z, i\right)}, \\
\beta_{n r \rightarrow k m_{k}}^{\left(q_{a}, z, i\right)}= & \sigma^{2}+\sum_{k^{\prime} m_{k}^{\prime} \in \mathcal{C}_{n r}} \sum_{q_{a}^{\prime} \in m_{k}^{\prime}}\left(\left|h_{k^{\prime}, q_{a}^{\prime}, n}^{(r)}\right|^{2} \omega_{k^{\prime} m_{k}^{\prime} \rightarrow n r}^{\left(q_{a}^{\prime}, z, i\right)}\right) \\
& -\left|h_{k, q_{a}, n}^{(r)}\right|^{2} \omega_{k m_{k} \rightarrow n r}^{\left(q_{a}, z, i\right)},
\end{aligned}
$$

respectively.

3) $C N$ update: The updated $\delta_{n r \rightarrow k m_{k}}^{(i)}\left(t_{k, q_{a}}^{(z)}\right)$ will be conveyed to $\mathrm{CN} n r$ via the edge $\bar{e}_{k m_{k}, n r}$ of the $3 \mathrm{D}$ factor graph, for updating $\eta_{k, n}^{(q, i)}$. Following the message passing rules, we have

$$
\eta_{k m_{k} \rightarrow n r}^{(i)}\left(t_{k, q_{a}}^{(z)}\right)=\varepsilon_{k m_{k}, n r} \prod_{n^{\prime} r^{\prime} \in \mathcal{U}_{k m_{k}} \backslash n r} \delta_{n^{\prime} r^{\prime} \rightarrow k m_{k}}^{(i)}\left(t_{k, q_{a}}^{(z)}\right),
$$

where $\varepsilon_{k m_{k}, n r}$ is the normalisation factor enabling $\sum_{m_{k} \in \mathcal{M}_{1}} \sum_{t_{k, q_{a}} \in \tilde{\mathcal{S}}} \eta_{k m_{k} \rightarrow n r}^{(i)}\left(t_{k, q_{a}}^{(z)}\right)=1$. By applying (22b) to (25), we obtain

$$
\begin{aligned}
& \eta_{k m_{k} \rightarrow n r}^{(i)}\left(t_{k, q_{a}}^{(z)}\right) \\
& =\frac{\mathcal{N}_{\mathbb{C}}\left(t_{k, q_{a}}^{(z)} ; \lambda_{k m_{k} \rightarrow n r}^{\left(q_{a}, z, i\right)}, \varphi_{k m_{k} \rightarrow n r}^{\left(q_{a}, z, i\right)}\right)}{\sum_{m_{k}^{\prime} \in \mathcal{M}_{1}} \sum_{t_{k, q_{a}}^{\prime(z)} \in \tilde{\mathcal{S}}} \mathcal{N}_{\mathbb{C}}\left(t_{k, q_{a}}^{(z)} ; \lambda_{k m_{k}^{\prime} \rightarrow n r}^{\left(q_{a}, z, i\right)}, \varphi_{k m_{k}^{\prime} \rightarrow n r}^{\left(q_{a}, z, i\right)}\right)},
\end{aligned}
$$


where $\varphi_{k m_{k} \rightarrow n r}^{\left(q_{a}, z, i\right)}$ and $\lambda_{k m_{k} \rightarrow n r}^{\left(q_{a}, z, i\right)}$ can be expressed respectively as

$$
\begin{aligned}
& \varphi_{k m_{k} \rightarrow n r}^{\left(q_{a}, z, i\right)}=\lambda_{k m_{k} \rightarrow n r}^{\left(q_{a}, z, i\right)} \\
& \times\left(\sum_{n^{\prime} r^{\prime} \in \mathcal{U}_{k m_{k}}} \frac{h_{k, q_{a}, n^{\prime}}^{*\left(r^{\prime}\right)} \alpha_{n^{\prime} r^{\prime} \rightarrow k m_{k}}^{\left(q_{a}, z, i\right)}}{\beta_{n^{\prime} r^{\prime} \rightarrow k m_{k}}^{\left(q_{a}, z, i\right)}}-\frac{h_{k, q_{a}, n}^{*(r)} \alpha_{n r \rightarrow k m_{k}}^{\left(q_{a}, z, i\right)}}{\beta_{n r \rightarrow k m_{k}}^{\left(q_{a}, z, i\right)}}\right) \\
& \lambda_{k m_{k} \rightarrow n r}^{\left(q_{a}, z, i\right)}=\left(\sum_{n^{\prime} r^{\prime} \in \mathcal{U}_{k m_{k}}} \frac{\left|h_{k, q_{a}, n^{\prime}}^{\left(r^{\prime}\right)}\right|^{2}}{\beta_{n^{\prime} r^{\prime} \rightarrow k m_{k}}^{\left(q_{a}, z, i\right)}}-\frac{\left|h_{k, q_{a}, n}^{(r)}\right|^{2}}{\beta_{n r \rightarrow k m_{k}}^{\left(q_{a}, z, i\right)}}\right)^{-1}
\end{aligned}
$$

A classic technique of finding the Gaussian approximation of $\eta_{k m_{k} \rightarrow n r}^{(i)}\left(t_{k, q_{a}}^{(z)}\right)$ in the $i$-th iteration is by minimizing the inclusive Kullback-Leibler (KL) divergence [39,41], expressed as $\operatorname{KL}\left(\eta_{k m_{k} \rightarrow n r}^{(i)}\left(t_{k, q_{a}}^{(z)}\right) \| \tilde{\eta}_{k m_{k} \rightarrow n r}^{(i)}\left(t_{k, q_{a}}^{(z)}\right)\right)$, giving the mean and variance of

$$
\begin{aligned}
& \mu_{k m_{k} \rightarrow n r}^{\left(q_{a}, z, i\right)}=\sum_{t_{k, q_{a}}^{(z)} \in \tilde{\mathcal{S}}^{(z)}} t_{k, q_{a}}^{(z)} \cdot \eta_{k m_{k} \rightarrow n r}^{(i-1)}\left(t_{k, q_{a}}^{(z)}\right) \\
& \omega_{k m_{k} \rightarrow n r}^{\left(q_{a}, z, i\right)}=\sum_{t_{k, q_{a}}^{(z)} \in \tilde{\mathcal{S}}}\left|t_{k, q_{a}}^{(z)}\right|^{2} \cdot \eta_{k m_{k} \rightarrow n r}^{(i-1)}\left(t_{k, q_{a}}^{(z)}\right)-\left|\mu_{k m_{k} \rightarrow n r}^{\left(q_{a}, z, i\right)}\right|^{2} .
\end{aligned}
$$

Furthermore, since STC is employed in the proposed STC/GSM-SCDMA system, the QAM symbol $s_{k, q_{a}}^{(1)}$ of user $k$, which is initially transmitted by the $q_{a}$-th TA, is also retransmitted by the other activated TAs in the following $(Z-1)$ SDs, after some form of 'STC-transformation', for example, in its additive inverse or conjugate form, as discussed in Section II-A. Hence, the information associated with $s_{k, q_{a}}^{(1)}$ can be obtained over all the $Z$ SDs and can be exploited for improving the reliability of the information passing in the next iteration. For example, when Alamouti's code of (1) is employed, the mean $\varphi_{k m_{k} \rightarrow n r}^{\left(q_{a}, z, i\right)}$ of (27) and variance $\lambda_{k m_{k} \rightarrow n r}^{\left(q_{a}, z, i\right)}$ of (28) for $z=1,2$, and $q_{a}=q_{1}, q_{a}=q_{2}$ can be expressed in (31) and (32), respectively.

Considering (31) as an example, we combine the statistics of the QAM symbol $s_{k, q_{1}}$ over the $Z=2$ SDs. Since $s_{k}^{2}$ is transmitted by the $q_{1}$-th TA in the 1 -st SD and its conjugate form $s_{k, q_{1}}^{*}$ is transmitted by $q_{2}$-th TA in the 2 nd SD, the mean $\alpha_{n r \rightarrow k m_{r}}^{\left(q_{2}, 2, i\right)}$ of the Gaussian distribution $\mathcal{N}_{\mathbb{C}}\left(h_{k, q_{2}, n}^{(r)} t_{k, q_{2}}^{(2)} ; \alpha_{n r \rightarrow k m_{k}}^{\left(q_{2}, 2, i\right)}, \beta_{n r \rightarrow k m_{k}}^{\left(q_{2}, 2, i\right)}\right)$ has to be conjugated before combining, giving the expression of the second term of (31).

4) Final iteration: After a pre-determined number $\bar{I}$ of iterations is reached, we calculate the information $\eta_{k m_{k}}^{(\bar{I})}\left(t_{k, q_{a}}^{(z)}\right)$ of the $k m_{k}$-th $\mathrm{VN}$ gleaned from all CNs that are connected to the $k m_{k}$-th $\mathrm{VN}$, which can be expressed as

$$
\begin{aligned}
& \eta_{k m_{k}}^{(\bar{I})}\left(t_{k, q_{a}}^{(z)}\right) \\
& =\frac{\mathcal{N}_{\mathbb{C}}\left(t_{k, q_{a}}^{(z)} ; \lambda_{k m_{k}}^{\left(q_{a}, z, \bar{I}\right)}, \varphi_{k m_{k}}^{\left(q_{a}, z, \bar{I}\right)}\right)}{\sum_{m_{k}^{\prime} \in \mathcal{M}_{1}} \sum_{t_{k, q_{a}}^{\prime}(z)} \in \tilde{\mathcal{S}} \mathcal{N}_{\mathbb{C}}\left(t_{k, q_{a}}^{(z)} ; \lambda_{k m_{k}^{\prime}}^{\left(q_{a}, z, \bar{I}\right)}, \varphi_{k m_{k}^{\prime}}^{\left(q_{a}, z, \bar{I}\right)}\right)},
\end{aligned}
$$

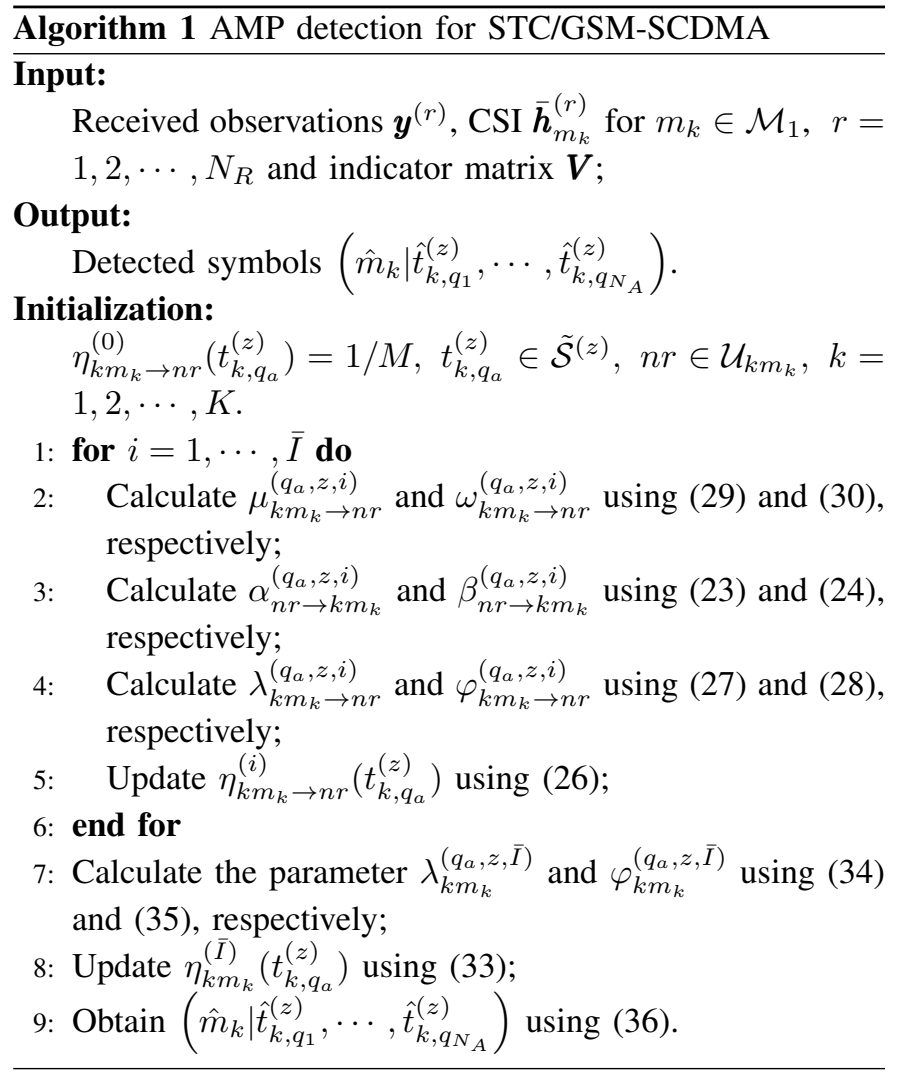

where $\lambda_{k m_{k}}^{\left(q_{a}, z, \bar{I}\right)}$ and $\varphi_{k m_{k}}^{\left(q_{a}, z, \bar{I}\right)}$ are the mean and variance of the variable $t_{k, q_{a}}^{(z)}$ in the $\bar{I}$-th iteration and can be respectively expressed as

$$
\begin{aligned}
& \varphi_{k m_{k}}^{\left(q_{a}, z, \bar{I}\right)}=\lambda_{k m_{k}}^{\left(q_{a}, z, I\right)}\left(\sum_{n^{\prime} r^{\prime} \in \mathcal{U}_{k m_{k}}} \frac{h_{k, q_{a}, n^{\prime}}^{*\left(r^{\prime}\right)} \alpha_{n^{\prime} r^{\prime}}^{\left(q_{a}, z, \bar{I}\right)}}{\beta_{n^{\prime} r^{\prime} \rightarrow k m_{k}}^{\left(q_{a}, z, I\right)}}\right) \\
& \lambda_{k m_{k}}^{\left(q_{a}, z, \bar{I}\right)}=\left(\sum_{n^{\prime} r^{\prime} \in \mathcal{U}_{k m_{k}}} \frac{\left|h_{k, q_{a}, n^{\prime}}^{\left(r^{\prime}\right)}\right|^{2}}{\beta_{n^{\prime} r^{\prime}}^{\left(q_{a}, z, I\right)}}\right)^{-1} .
\end{aligned}
$$

5) Symbol mapping: The symbol mapping process can be expressed as

$$
\begin{aligned}
\hat{m}_{k} \mid \hat{t}_{k, q_{1}}^{(z)}, \cdots, \hat{t}_{k, q_{N_{A}}}^{(z)}= & \arg \max _{\substack{t_{k, q_{1}}^{(z)}, \cdots, t_{k, q_{N}}^{(z)} \\
m_{k} \in \mathcal{\mathcal { S }}^{(z)}}} \prod_{a=1}^{N_{A}} \eta_{k m_{k}}^{(\bar{I})}\left(t_{k, q_{a}}^{(z)}\right), \\
& k=1,2, \ldots, K
\end{aligned}
$$

In summary, the proposed AMP detection algorithm of our STC/GSM-SCDMA system is summarized in Algorithm 1 and the complexity of the proposed AMP detector as well as its comparison to the MPA detector will be discussed in Section V-B.

Note that our proposed detectors can be directly applied to the STC/GSM-SCDMA downlink, where the BS transmits the superposition of SCMA symbols for $K$ users after STC/GSM processing by using $N$ subcarriers. At the receiver side, each user can simultaneously and independently detect the signal by MPA or AMP detection based on the factor graphs of Fig. 2 or 3 , respectively. 


$$
\begin{aligned}
& \varphi_{k m_{k} \rightarrow n r}^{\left(q_{1}, 1, i\right)}=\lambda_{k m_{k} \rightarrow n r}^{\left(q_{1}, 1, i\right)}\left(\sum_{n^{\prime} r^{\prime} \in \mathcal{U}_{k m_{k}}} \frac{h_{k, q_{1}, n^{\prime}}^{*\left(r^{\prime}\right)} \alpha_{n^{\prime} r^{\prime} \rightarrow k m_{k}}^{\left(q_{1}, 1, i\right)}}{\beta_{n^{\prime} r^{\prime} \rightarrow k m_{k}}^{\left(q_{1}, 1, i\right)}}+\sum_{n^{\prime} r^{\prime} \in \mathcal{U}_{k m_{k}}} \frac{h_{k, q_{2}, n^{\prime}}^{*\left(r^{\prime}\right)} \alpha_{n^{\prime} r^{\prime} \rightarrow k m_{k}}^{*\left(q_{2}, 2, i\right)}}{\beta_{n^{\prime} r^{\prime} \rightarrow k m_{k}}^{\left(q_{2}, 2, i\right)}}-\frac{h_{k, q_{1}, n}^{*(r)} \alpha_{n r \rightarrow k m_{k}}^{\left(q_{1}, 1, i\right)}}{\beta_{n r \rightarrow k m_{k}}^{\left(q_{1}, 1, i\right)}}\right) \\
& \varphi_{k m_{k} \rightarrow n r}^{\left(q_{2}, 1, i\right)}=\lambda_{k m_{k} \rightarrow n r}^{\left(q_{2}, 1, i\right)}\left(\sum_{n^{\prime} r^{\prime} \in \mathcal{U}_{k m_{k}}} \frac{h_{k, q_{2}, n^{\prime}}^{*\left(r^{\prime}\right)} \alpha_{n^{\prime} r^{\prime} \rightarrow k m_{k}}^{\left(q_{2}, 1, i\right)}}{\beta_{n^{\prime} r^{\prime} \rightarrow k m_{k}}^{\left(q_{2}, 1, i\right)}}-\sum_{n^{\prime} r^{\prime} \in \mathcal{U}_{k m_{k}}} \frac{h_{k, q_{1}, n^{\prime}}^{*\left(r^{\prime}\right)} \alpha_{n^{\prime} r^{\prime} \rightarrow k m_{k}}^{*\left(q_{1}, 2, i\right)}}{\beta_{n^{\prime} r^{\prime} \rightarrow k m_{k}}^{\left(q_{1}, 2, i\right)}}-\frac{h_{k, q_{2}, n}^{*(r)} \alpha_{n r \rightarrow k m_{k}}^{\left(q_{2}, 1, i\right)}}{\beta_{n r \rightarrow k m_{k}}^{\left(q_{2}, 1, i\right)}}\right) \\
& \varphi_{k m_{k} \rightarrow n r}^{\left(q_{1}, 2, i\right)}=\lambda_{k m_{k} \rightarrow n r}^{\left(q_{1}, 2, i\right)}\left(\sum_{n^{\prime} r^{\prime} \in \mathcal{U}_{k m_{k}}} \frac{h_{k, q_{1}, n^{\prime}}^{*\left(r^{\prime}\right)} \alpha_{n^{\prime} r^{\prime} \rightarrow k m_{k}}^{\left(q_{1}, 2, i\right)}}{\beta_{n^{\prime} r^{\prime} \rightarrow k m_{k}}^{\left(q_{1}, 2, i\right)}}+\sum_{n^{\prime} r^{\prime} \in \mathcal{U}_{k m_{k}}} \frac{h_{k, q_{2}, n^{\prime}}^{*\left(r^{\prime}\right)} \alpha_{n^{\prime} r^{\prime} \rightarrow k m_{k}}^{*\left(q_{2}, 1, i\right)}}{\beta_{n^{\prime} r^{\prime} \rightarrow k m_{k}}^{\left(q_{2}, 1, i\right)}}-\frac{h_{k, q_{1}, n}^{*(r)} \alpha_{n r \rightarrow k m_{k}}^{\left(q_{1}, 2, i\right)}}{\beta_{n r \rightarrow k m_{k}}^{\left(q_{2}, 1, i\right)}}\right) \\
& \varphi_{k m_{k} \rightarrow n r}^{\left(q_{2}, 2, i\right)}=\lambda_{k m_{k} \rightarrow n r}^{\left(q_{2}, 2, i\right)}\left(\sum_{n^{\prime} r^{\prime} \in \mathcal{U}_{k m_{k}}} \frac{h_{k, q_{2}, n^{\prime}}^{*\left(r^{\prime}\right)} \alpha_{n^{\prime} r^{\prime} \rightarrow k m_{k}}^{\left(q_{2}, 2, i\right)}}{\beta_{n^{\prime} r^{\prime} \rightarrow k m_{k}}^{\left(q_{2}, 2, i\right)}}+\sum_{n^{\prime} r^{\prime} \in \mathcal{U}_{k m_{k}}} \frac{h_{k, q_{1}, n^{\prime}}^{*\left(r^{\prime}\right)} \alpha_{n^{\prime} r^{\prime} \rightarrow k m_{k}}^{*\left(q_{1}, 1, i\right)}}{\beta_{n^{\prime} r^{\prime} \rightarrow k m_{k}}^{\left(q_{1}, 1, i\right)}}-\frac{h_{k, q_{2}, n}^{*(r)} \alpha_{n r \rightarrow k m_{k}}^{\left(q_{2}, 2, i\right)}}{\beta_{n r \rightarrow k m_{k}}^{\left(q_{2}, 1, i\right)}}\right) \\
& \lambda_{k m_{k} \rightarrow n r}^{\left(q_{1}, 1, i\right)}=\left(\sum_{n^{\prime} r^{\prime} \in \mathcal{U}_{k m_{k}}} \frac{\left|h_{k, q_{1}, n^{\prime}}^{\left(r^{\prime}\right)}\right|^{2}}{\beta_{n^{\prime} r^{\prime} \rightarrow k m_{k}}^{\left(q_{1}, 1, i\right)}}+\sum_{n^{\prime} r^{\prime} \in \mathcal{U}_{k m_{k}}} \frac{\left|h_{k, q_{2}, n^{\prime}}^{\left(r^{\prime}\right)}\right|^{2}}{\beta_{n^{\prime} r^{\prime} \rightarrow k m_{k}}^{\left(q_{2}, i\right)}}-\frac{\left|h_{k, q_{1}, n}^{(r)}\right|^{2}}{\beta_{n r \rightarrow k m_{k}}^{\left(q_{1}, 1, i\right)}}\right)^{-1} \\
& \lambda_{k m_{k} \rightarrow n r}^{\left(q_{2}, 1, i\right)}=\left(\sum_{n^{\prime} r^{\prime} \in \mathcal{U}_{k m_{k}}} \frac{\left|h_{k, q_{2}, n^{\prime}}^{\left(r^{\prime}\right)}\right|^{2}}{\beta_{n^{\prime} r^{\prime} \rightarrow k m_{k}}^{\left(q_{2}, 1, i\right)}}+\sum_{n^{\prime} r^{\prime} \in \mathcal{U}_{k m_{k}}} \frac{\left|h_{k, q_{1}, n^{\prime}}^{\left(r^{\prime}\right)}\right|^{2}}{\beta_{n^{\prime} r^{\prime} \rightarrow k m_{k}}^{\left(q_{1}, 2, i\right)}}-\frac{\left|h_{k, q_{2}, n}^{(r)}\right|^{2}}{\beta_{n r \rightarrow k m_{k}}^{\left(q_{2}, 1, i\right)}}\right)^{-1} \\
& \lambda_{k m_{k} \rightarrow n r}^{\left(q_{1}, 2, i\right)}=\left(\sum_{n^{\prime} r^{\prime} \in \mathcal{U}_{k m_{k}}} \frac{\left|h_{k, q_{1}, n^{\prime}}^{\left(r^{\prime}\right)}\right|^{2}}{\beta_{n^{\prime} r^{\prime} \rightarrow k m_{k}}^{\left(q_{1}, 2, i\right)}}+\sum_{n^{\prime} r^{\prime} \in \mathcal{U}_{k m_{k}}} \frac{\left|h_{k, q_{2}, n^{\prime}}^{\left(r^{\prime}\right)}\right|^{2}}{\beta_{n^{\prime} r^{\prime} \rightarrow k m_{k}}^{\left(q_{2}, 1, i\right)}}-\frac{\left|h_{k, q_{1}, n}^{(r)}\right|^{2}}{\beta_{n r \rightarrow k m_{k}}^{\left(q_{1}, 2, i\right)}}\right)^{-1} \\
& \lambda_{k m_{k} \rightarrow n r}^{\left(q_{2}, 2, i\right)}=\left(\sum_{n^{\prime} r^{\prime} \in \mathcal{U}_{k m_{k}}} \frac{\left|h_{k, q_{2}, n^{\prime}}^{\left(r^{\prime}\right)}\right|^{2}}{\beta_{n^{\prime} r^{\prime} \rightarrow k m_{k}}^{\left(q_{2}, 2, i\right)}}+\sum_{n^{\prime} r^{\prime} \in \mathcal{U}_{k m_{k}}} \frac{\left|h_{k, q_{1}, n^{\prime}}^{\left(r^{\prime}\right)}\right|^{2}}{\beta_{n^{\prime} r^{\prime} \rightarrow k m_{k}}^{\left(q_{1}, 1, i\right)}}-\frac{\left|h_{k, q_{2}, n}^{(r)}\right|^{2}}{\beta_{n r \rightarrow k m_{k}}^{\left(q_{2}, 2, i\right)}}\right)^{-1}
\end{aligned}
$$

\section{Single-User Performance Analysis}

In this section, we analyse the single-user performance bound as the benchmark of our proposed STC/GSM-SCDMA system for transmission over frequency selective channels. When the proposed STC/GSM-SCDMA system supports only a single user, the received observations of (9) become

$$
\boldsymbol{Y}=\frac{1}{\sqrt{Z}} \boldsymbol{H} \boldsymbol{E}_{m} \boldsymbol{T}+\boldsymbol{n} .
$$

Then, following [26], the pairwise error probability (PEP) when the transmitted $\boldsymbol{E}_{m} \boldsymbol{T}$ is detected as $\boldsymbol{E}_{\tilde{m}} \tilde{\boldsymbol{T}} \neq \boldsymbol{E}_{m} \boldsymbol{T}$ can be expressed as

$$
P\left(\boldsymbol{E}_{m} \boldsymbol{T} \rightarrow \boldsymbol{E}_{\tilde{m}} \tilde{\boldsymbol{T}}\right)=E_{\boldsymbol{H}}\left[Q\left(\sqrt{\frac{\gamma}{2}\left\|\boldsymbol{H} \boldsymbol{E}_{\tilde{m}} \tilde{\boldsymbol{T}}-\boldsymbol{H} \boldsymbol{E}_{m} \boldsymbol{T}\right\|}\right)\right],
$$

where the $Q$-function $Q(x)$ is defined as $Q(x)=$ $(2 \pi)^{-1 / 2} \int_{x}^{\infty} e^{-t^{2} / 2} d t$, and $E_{\boldsymbol{H}}[\cdot]$ denotes the average with respect to the channels between the user and the BS. Upon invoking the alternative representation of $Q(x)$ of $Q(x)=$ $\pi^{-1} \int_{0}^{\pi / 2} \exp \left(-\frac{x^{2}}{2 \sin ^{2} \theta}\right) d \theta$ [42], the PEP of (38) can be expressed as

$$
\begin{aligned}
& P\left(\boldsymbol{E}_{m} \boldsymbol{T} \rightarrow \boldsymbol{E}_{\tilde{m}} \tilde{\boldsymbol{T}}\right) \\
& =E_{\boldsymbol{H}}\left[\frac{1}{\pi} \int_{0}^{\frac{\pi}{2}} \exp \left(-\frac{\gamma}{4 \sin ^{2} \theta}\left\|\boldsymbol{H} \boldsymbol{E}_{\tilde{m}} \tilde{\boldsymbol{T}}-\boldsymbol{H} \boldsymbol{E}_{m} \boldsymbol{T}\right\|^{2}\right) d \theta\right] \\
& =\frac{1}{\pi} \int_{0}^{\frac{\pi}{2}} \Phi_{\left\|\boldsymbol{H} \boldsymbol{E}_{\tilde{m}} \tilde{\boldsymbol{T}}-\boldsymbol{H} \boldsymbol{E}_{m} \boldsymbol{T}\right\|^{2}}\left(-\frac{\gamma}{4 \sin ^{2} \theta}\right) d \theta
\end{aligned}
$$

where $\Phi_{\left\|\boldsymbol{H} \boldsymbol{E}_{\tilde{m}} \tilde{\boldsymbol{T}}-\boldsymbol{H} \boldsymbol{E}_{m} \boldsymbol{T}\right\|^{2}}(\cdot)$ is the moment generation function (MGF) of the variable $\left\|\boldsymbol{H} \boldsymbol{E}_{\tilde{m}} \tilde{\boldsymbol{T}}-\boldsymbol{H} \boldsymbol{E}_{m} \boldsymbol{T}\right\|^{2}$.

In the STC/GSM-SCDMA system, errors may optionally corrupt only the GSM symbol, only the QAM symbols, or both of them. When considering these three cases separately, the corresponding PEP $P\left(\boldsymbol{E}_{m} \boldsymbol{T} \rightarrow \boldsymbol{E}_{\tilde{m}} \tilde{\boldsymbol{T}}\right)$ of (39b) can be derived as follows.

\section{1) Errors in GSM Symbol Only}

When only the GSM symbol is erroneous, i.e. $\boldsymbol{E}_{\tilde{m}} \neq$ $\boldsymbol{E}_{m}$ and $\tilde{\boldsymbol{T}}=\boldsymbol{T}$, the PEP $P_{1}\left(\boldsymbol{E}_{m} \boldsymbol{T} \rightarrow \boldsymbol{E}_{\tilde{m}} \boldsymbol{T}\right)$ can be 
expressed as

$$
\begin{aligned}
& P_{1}\left(\boldsymbol{E}_{m} \boldsymbol{T} \rightarrow \boldsymbol{E}_{\tilde{m}} \boldsymbol{T}\right) \\
= & E_{\boldsymbol{H}}\left[\frac{1}{\pi} \int_{0}^{\frac{\pi}{2}} \exp \left(-\frac{\gamma\|\boldsymbol{T}\|^{2}}{4 \sin ^{2} \theta}\left\|\boldsymbol{H} \boldsymbol{E}_{\tilde{m}}-\boldsymbol{H} \boldsymbol{E}_{m}\right\|^{2}\right) d \theta\right] \\
= & E_{\boldsymbol{H}}\left[\frac{1}{\pi} \int_{0}^{\frac{\pi}{2}} \exp \left(-\frac{\gamma\|\boldsymbol{T}\|^{2}}{4 \sin ^{2} \theta}\left\|\boldsymbol{H} \boldsymbol{\Omega}_{1}\right\|^{2}\right) d \theta\right] \\
= & \frac{1}{\pi} \int_{0}^{\frac{\pi}{2}} \Phi_{\left\|\boldsymbol{H} \boldsymbol{\Omega}_{1}\right\|^{2}}\left(-\frac{\|\boldsymbol{T}\|^{2} \gamma}{4 \sin ^{2} \theta}\right) d \theta
\end{aligned}
$$

where $\Omega_{1}=\boldsymbol{E}_{\tilde{m}}-\boldsymbol{E}_{m}$.

2) Errors in QAM Symbols Only

By contrast, when the errors corrupt only the QAM symbol(s), we have $\tilde{\boldsymbol{T}} \neq \boldsymbol{T}$ and $\boldsymbol{E}_{\tilde{m}}=\boldsymbol{E}_{m}$. Then the PEP of (39b) can re-written as

$$
\begin{aligned}
& P_{2}\left(\boldsymbol{E}_{m} \boldsymbol{T} \rightarrow \boldsymbol{E}_{m} \tilde{\boldsymbol{T}}\right) \\
= & E_{\boldsymbol{H}}\left[\frac{1}{\pi} \int_{0}^{\frac{\pi}{2}} \exp \left(-\frac{\gamma}{4 \sin ^{2} \theta}\left\|\boldsymbol{H} \boldsymbol{E}_{m} \tilde{\boldsymbol{T}}-\boldsymbol{H} \boldsymbol{E}_{m} \boldsymbol{T}\right\|^{2}\right) d \theta\right] \\
= & E_{\boldsymbol{H}}\left[\frac{1}{\pi} \int_{0}^{\frac{\pi}{2}} \exp \left(-\frac{\|\tilde{\boldsymbol{T}}-\boldsymbol{T}\|^{2} \gamma}{4 \sin ^{2} \theta}\left\|\boldsymbol{H} \boldsymbol{E}_{s_{1}}\right\|^{2}\right) d \theta\right] \\
= & \frac{1}{\pi} \int_{0}^{\frac{\pi}{2}} \Phi_{\left\|\boldsymbol{H} \boldsymbol{\Omega}_{2}\right\|^{2}}\left(-\frac{\|\tilde{\boldsymbol{T}}-\boldsymbol{T}\|^{2} \gamma}{4 \sin ^{2} \theta}\right) d \theta
\end{aligned}
$$

where $\boldsymbol{\Omega}_{2}=\boldsymbol{E}_{m}$.

\section{3) Errors in both GSM and QAM Symbols}

Finally, when the errors occur in both the GSM and QAM symbol(s), we have $\boldsymbol{E}_{\tilde{m}} \neq \boldsymbol{E}_{m}$ and $\tilde{\boldsymbol{T}} \neq \boldsymbol{T}$. Correspondingly, (39b) can be modified to

$$
\begin{aligned}
& P_{3}\left(\boldsymbol{E}_{m} \boldsymbol{T} \rightarrow \boldsymbol{E}_{\tilde{m}} \tilde{\boldsymbol{T}}\right) \\
& =E_{\boldsymbol{H}}\left[\frac{1}{\pi} \int_{0}^{\frac{\pi}{2}} \exp \left(-\frac{\gamma}{4 \sin ^{2} \theta}\left\|\boldsymbol{H} \boldsymbol{E}_{\tilde{m}} \tilde{\boldsymbol{T}}-\boldsymbol{H} \boldsymbol{E}_{m} \boldsymbol{T}\right\|^{2}\right) d \theta\right] \\
& =E_{\boldsymbol{H}}\left[\frac{1}{\pi} \int_{0}^{\frac{\pi}{2}} \exp \left(-\frac{\gamma}{4 \sin ^{2} \theta}\left\|\boldsymbol{H} \boldsymbol{\Omega}_{3}\right\|^{2}\right) d \theta\right] \\
& =\frac{1}{\pi} \int_{0}^{\frac{\pi}{2}} \Phi_{\left\|\boldsymbol{H} \boldsymbol{\Omega}_{3}\right\|^{2}}\left(-\frac{\gamma}{4 \sin ^{2} \theta}\right) d \theta
\end{aligned}
$$

where $\boldsymbol{\Omega}_{3}=\boldsymbol{E}_{\tilde{m}} \tilde{\boldsymbol{T}}-\boldsymbol{E}_{m} \boldsymbol{T}$.

Since the channels experience correlated Rayleigh fading, the MGF of $\left\|\boldsymbol{H} \boldsymbol{\Omega}_{i}\right\|^{2}$ can be derived in the same way as that in $[33,43]$, which can be expressed as

$$
\Phi_{\left\|\boldsymbol{H} \boldsymbol{\Omega}_{i}\right\|^{2}}(t)=\operatorname{det}\left[\boldsymbol{I}_{N_{R} N Z}-t \boldsymbol{R}\left(\boldsymbol{I}_{N_{R} N} \otimes\left(\boldsymbol{\Omega}_{i} \boldsymbol{\Omega}_{i}^{H}\right)\right)\right]^{-1},
$$

where $\operatorname{det}(\cdot)$ denotes the determinant of a matrix and $R$ is the $\left(N_{R} N Z \times N_{R} N Z\right)$ covariance matrix of $\operatorname{vec}\left(\boldsymbol{H}^{T}\right)$, where $\operatorname{vec}(\cdot)$ represents the vectorization operation of a matrix, converting the columns of a matrix into a single column vector. Note that we omit the calculations of $\boldsymbol{R}$, since it has been demonstrated in $[33,37]$.

If we substitute $\Phi_{\left\|\boldsymbol{H} \boldsymbol{\Omega}_{i}\right\|^{2}}(t)$ of (40)-(42) with (43) into (40), (41) and (42), respectively, the PEPs of (40), (41) and (42) representing the three erroneous scenarios can be re-written respectively as

$$
\begin{aligned}
& P_{1}\left(\boldsymbol{E}_{m} \boldsymbol{T} \rightarrow \boldsymbol{E}_{\tilde{m}} \boldsymbol{T}\right) \\
& =\frac{1}{\pi} \int_{0}^{\frac{\pi}{2}} \operatorname{det}\left[\boldsymbol{I}_{M_{1} U N}+\frac{\|\boldsymbol{T}\|^{2} \gamma}{4 \sin ^{2} \theta} \boldsymbol{R}\left(\boldsymbol{I}_{U N} \otimes\left(\boldsymbol{\Omega}_{1} \boldsymbol{\Omega}_{1}^{H}\right)\right)\right]^{-1} d \theta
\end{aligned}
$$

$$
\begin{aligned}
& P_{2}\left(\boldsymbol{E}_{m} \boldsymbol{T} \rightarrow \boldsymbol{E}_{m} \tilde{\boldsymbol{T}}\right) \\
& =\frac{1}{\pi} \int_{0}^{\frac{\pi}{2}} \operatorname{det}\left[\boldsymbol{I}_{M_{1} U N}+\frac{\|\tilde{\boldsymbol{T}}-\boldsymbol{T}\|^{2} \gamma}{4 \sin ^{2} \theta} \boldsymbol{R}\left(\boldsymbol{I}_{U N} \otimes\left(\boldsymbol{\Omega}_{2} \boldsymbol{\Omega}_{2}^{H}\right)\right)\right]^{-1} d \theta
\end{aligned}
$$

$$
\begin{aligned}
& P_{3}\left(\boldsymbol{E}_{m} \boldsymbol{T} \rightarrow \boldsymbol{E}_{\tilde{m}} \tilde{\boldsymbol{T}}\right) \\
& =\frac{1}{\pi} \int_{0}^{\frac{\pi}{2}} \operatorname{det}\left[\boldsymbol{I}_{M_{1} U N}+\frac{\gamma}{4 \sin ^{2} \theta} \boldsymbol{R}\left(\boldsymbol{I}_{U N} \otimes\left(\boldsymbol{\Omega}_{3} \boldsymbol{\Omega}_{3}^{H}\right)\right)\right]^{-1} d \theta .
\end{aligned}
$$

Consequently, following the analysis in [33] and considering the three PEPs, the upper bound of the average BER (ABER) $P_{e}$ of the single-user STC/GSM-SCDMA system can be expressed as

$$
\begin{aligned}
P_{e} \leq & \frac{1}{b M_{1} M_{2}^{N_{A}}} \sum_{m \in \mathcal{M}_{1}} \sum_{\boldsymbol{s} \in \mathcal{X}} \sum_{\tilde{m} \in \mathcal{M}_{1}} \sum_{\tilde{\boldsymbol{s}} \in \mathcal{S}} D\left(\boldsymbol{b}_{1}\left|\boldsymbol{b}_{2}, \tilde{\boldsymbol{b}}_{1}\right| \tilde{\boldsymbol{b}}_{2}\right) \\
& \times P\left(\boldsymbol{E}_{m} \boldsymbol{T} \rightarrow \boldsymbol{E}_{\tilde{m}} \tilde{\boldsymbol{T}}\right),
\end{aligned}
$$

where $D(\cdot, \cdot)$ is defined as the Hamming distance between two binary entries, and $\tilde{\boldsymbol{b}}_{i}, i=1,2$, represents the mistaken detection of the bit sequence $\boldsymbol{b}_{i}$.

\section{Performance Results}

In this section, the proposed STC/GSM-SCDMA system is characterized in terms of its BER vs. complexity in Sections $\mathrm{V}-\mathrm{A}$ and $\mathrm{V}-\mathrm{B}$, respectively.

\section{A. BER Performance}

First, we quantify the single-user bound of (45) for the proposed STC/GSM-SCDMA system, when communicating over the $L=1,2$ and 4 frequency-selective Rayleigh fading channels, as shown in Fig. 4, where the user spreads its signal over $d_{c}=2$ out of $N=16$ subcarriers, and $N_{T}=6, N_{A}=2, N_{R}=8$ or 16 is employed. The simulation results of the proposed STC/GSM-SCDMA system supporting a single user are also included in Fig. 4, where we can see that the theoretical bound and the simulation BER tend to converge upon increasing the SNR. Hence, we can conclude that when the SNR is sufficiently high so that a BER below $10^{-2}$ can be attained, the theoretical bound of (45) derived in Section IV can be employed as the single-user performance bound of our STC/GSM-SCDMA system, which provides design guidelines concerning the system parameters without the need for practical implementations.

Furthermore, we compare the BER performance of the proposed STC/GSM-SCDMA system to that of the popular MIMO-NOMA systems of $[31,33]$. More specifically, Fig. 5 


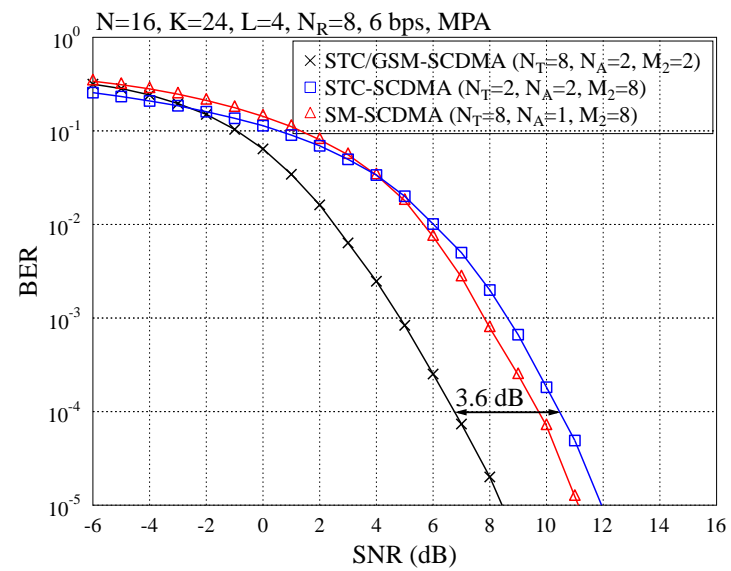

(a)

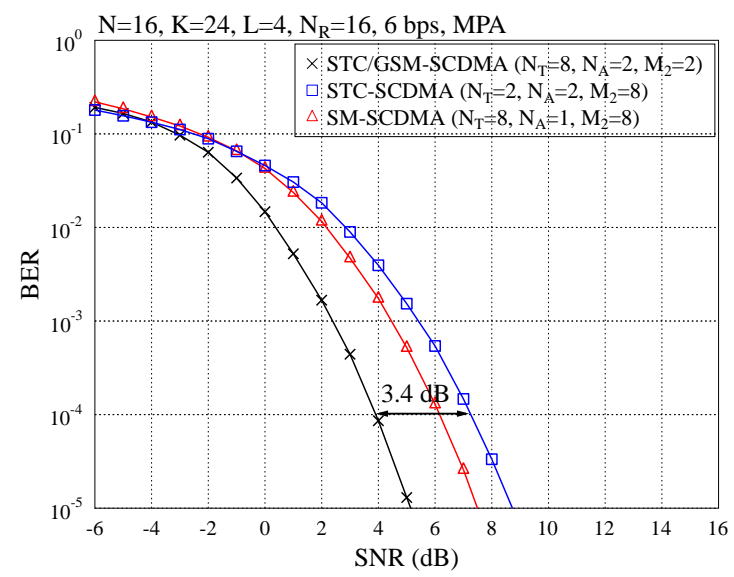

(b)

Fig. 5. BER performance of the SM-SCDMA [33], STC-SCMA [31] and the proposed STC/GSM-SCDMA systems supporting $K=24$ users using $N=16$ subcarriers when communicating over the $L=4$ frequency-selective Rayleigh fading channel at the same rate of 6 BPS, where all the considered systems employ the MPA detection, with (a) $N_{R}=8$ and (b) $N_{R}=16$.

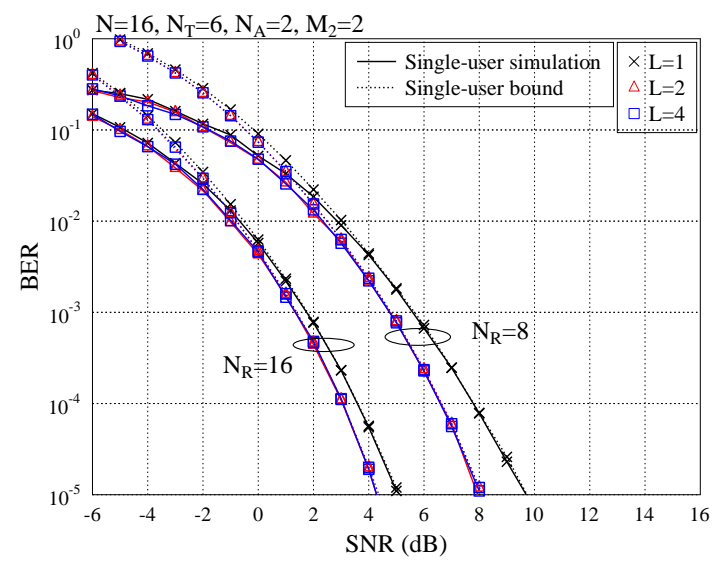

Fig. 4. Theoretical union bound of the proposed STC/GSM-SCDMA system and the simulated BER performance of the $N_{T}=6, N_{A}=2, N_{R}=4$ STC/GSM-SCDMA system supporting $K=1$ user over $L=1,2$ and 4 frequency-selective Rayleigh fading channels.

shows the BER performance of the SM-SCDMA [33], STCSCDMA [31] and the proposed STC/GSM-SCDMA systems supporting $K=24$ users employing $N=16$ subcarriers when communicating over the frequency-selective Rayleigh fading channels having $L=4$. Note that the design of $\boldsymbol{V}$ follows [44] for all $N=16$ systems. For fair comparison, all the systems considered transmit their signal at the same 6 BPS where all of them employ the MPA detector, associated with (a) $N_{R}=8$ and (b) $N_{R}=16$. We can see from both figures that our proposed STC/GSM-SCDMA system achieves superior BER performance, over the MIMO-NOMA benchmarks considered in Fig. 5, since our STC/GSM-SCDMA system exploits the diversity in the SFD and conveys the information bits both by the TA indices and by the QAM symbols.

Fig. 6 further investigates the BER performance of the proposed STC/GSM-SCDMA systems in demanding largescale access scenarios at a normalized user load of $150 \%$, where employing the MPA detector for communicating over

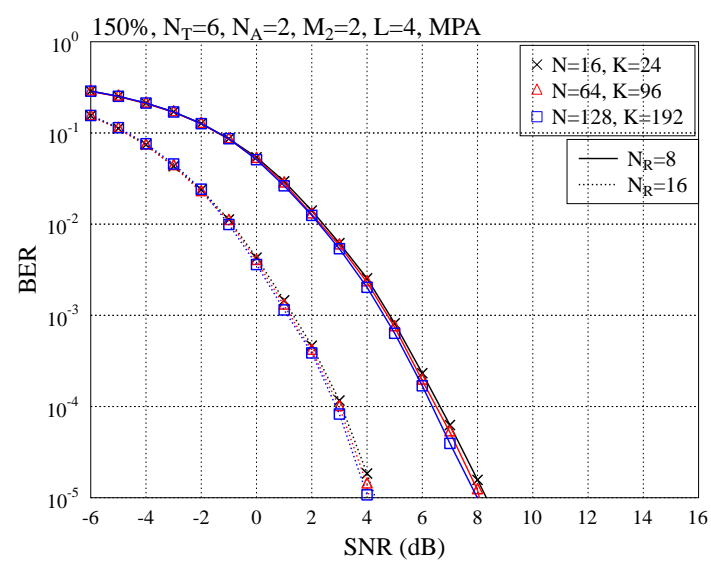

Fig. 6. BER performance of the proposed STC/GSM-SCDMA systems with $N_{T}=6, N_{A}=2, N_{R}=8$ or 16 employing the MPA detection with a normalized user load of $150 \%$ when communicating over the $L=4$ frequency-selective Rayleigh fading channel.

frequency-selective Rayleigh fading channel having $L=4$. Observe from Fig. 6 that the BER performance of different systems is rather similar as long as the normalized user load remains unchanged at $150 \%$. This is promising for demanding mMTC scenarios, where a BS may have to support thousands or even millions of devices.

Let us now demonstrate the influence of the normalized user load on the BER performance of the proposed STC/GSMSCDMA systems, as shown in Fig. 7. More specifically, in Fig. 7, we have $N_{T}=6$ TAs at each user's transmitter with $N_{A}=2$ TAs activated within a single $\mathrm{SD}$, supporting $K=16,24$ and 32 users by $N=16$ subcarriers, where $N_{R}=2,4,8$ or 16 RAs are employed. The single-user bound is included as the benchmarker. A slight performance loss can be observed at the demanding normalized user load of $200 \%$ in the low-SNR region. But when the BER is below $10^{-2}$ and the number of RAs is sufficiently high, the BER of our STC/GSMSCDMA system supporting $K=32$ users converges to that 


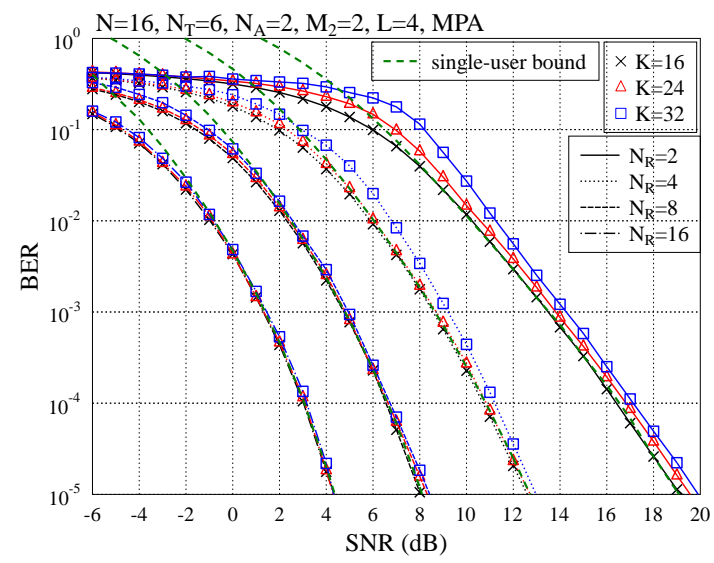

Fig. 7. BER performance of the proposed STC/GSM-SCDMA systems with $N=16, N_{T}=6, N_{A}=2, N_{R}=2,4,8$ or 16 employing the MPA detection with a normalized user load of $100 \%, 150 \%$ and $200 \%$ when communicating over the $L=4$ frequency-selective Rayleigh fading channel.

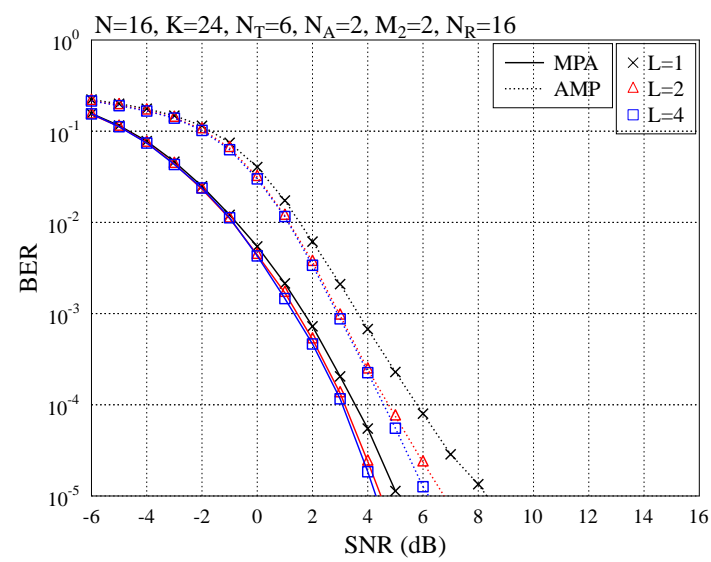

Fig. 8. BER performance of the $N_{T}=6, N_{A}=2, N_{R}=16$ STC/GSMSCDMA system employing the MPA detection and that employing AMP detection for supporting $K=24$ users by $N=16$ subcarriers, when communicating over the $L=1,2$ and 4 frequency-selective Rayleigh fading channels.

supporting $K=16$ users and even to the single-user bound Therefore, our STC/GSM-SCDMA system has the potential of supporting heavily-loaded NOMA systems in next-generation communications. It is also noticeable that in the case of $100 \%$ normalized user load, the MU BER performance is still upper bounded by the single-user bound.

The BER performance of the proposed low-complexity AMP detector of our STC/GSM-SCDMA system is shown in Fig. 8 and compared to the MPA detector, where $N_{T}=6$ TAs, and $N_{A}=2$ activated TAs are employed by each of the $K=16$ users and $N_{R}=16$ RAs are used by the BS. Again, $N=16$ subcarriers are employed to support $K=24$ users, when communicating over frequency-selective Rayleigh fading channels having $L=1,2$ and 4 . In the case of $L=4$, a $1.5 \mathrm{~dB}$ SNR loss can be observed from Fig. 8 at the BER of $10^{-3}$, when the AMP detection is employed, compared to that of the MPA detector. However, this is achieved at a potentially 1000 times lower detection complexity, which will be detailed

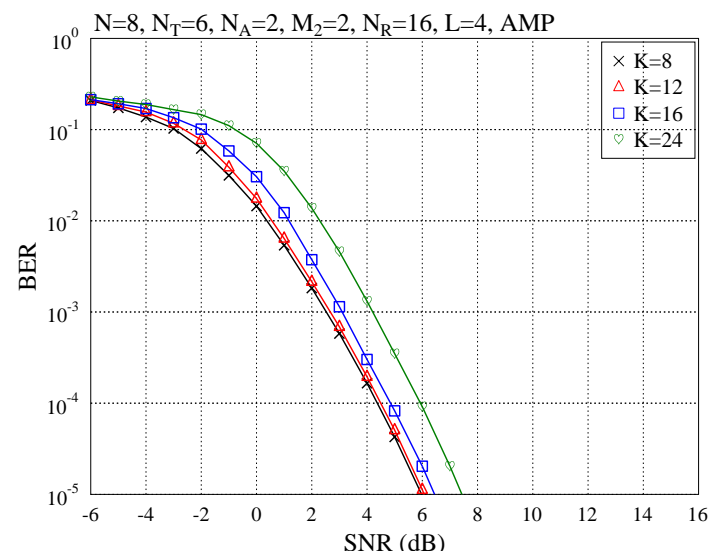

Fig. 9. BER performance of the $N_{T}=6, N_{A}=2, N_{R}=16$ STC/GSMSCDMA system employing AMP detection for supporting $K=8,12,16,24$ users by $N=8$ subcarriers, when communicating over the $L=4$ frequencyselective Rayleigh fading channel, where the design of $\boldsymbol{V}$ follows [45].

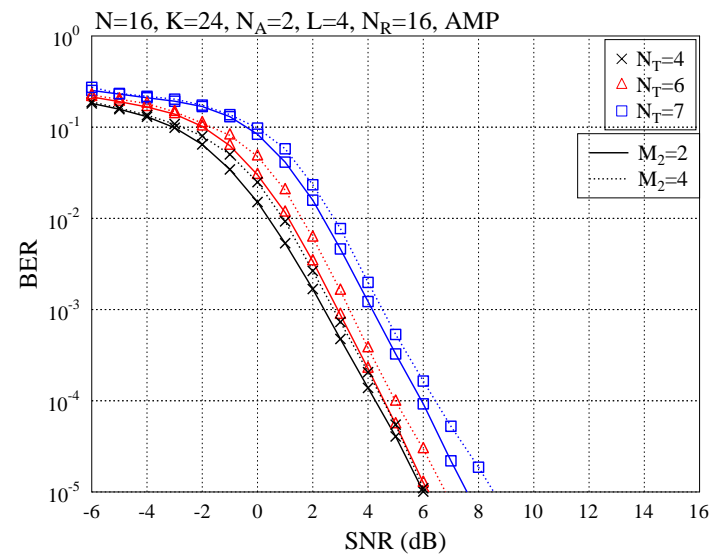

Fig. 10. BER performance of the $N_{A}=2, N_{R}=16$ STC/GSM-SCDMA systems employing AMP detection for supporting $K=24$ users by $N=16$ subcarriers, where each user is equipped with $N_{T}=4,6$ or 7 TAs, when communicating over the $L=4$ frequency-selective Rayleigh fading channel.

in Section V-B.

Fig. 9 characterizes the BER performance of the proposed STC/GSM-SCDMA systems supporting different overloaded systems when employing AMP detection, where $N_{T}=6$, $N_{A}=2, N_{R}=16$ and $N=8$. We can see from Fig. 9 that more significant performance loss can be observed at normalized user loads of $200 \%$ and $300 \%$. The reasons behind this are two fold. Firstly, the increasing number of users supported by the same number of subcarriers inevitably leads to more severe inter-user interference. Furthermore, the closed loops in the factor graph are unavoidable when the user load is higher than $150 \%$, which prevents the information from propagating through the entire factor graph.

The influence of $M_{1}$ and $M_{2}$ on the BER performance of our STC/GSM-SCDMA system is demonstrated in Fig. 10, where the $N_{R}=16$ STC/GSM-SCDMA system supports $K=$ 24 users by $N=16$ subcarriers and the AMP detector is employed. Firstly, it can be concluded that when the number of activated TAs is fixed, increasing $N_{T}$ tends to result in a 


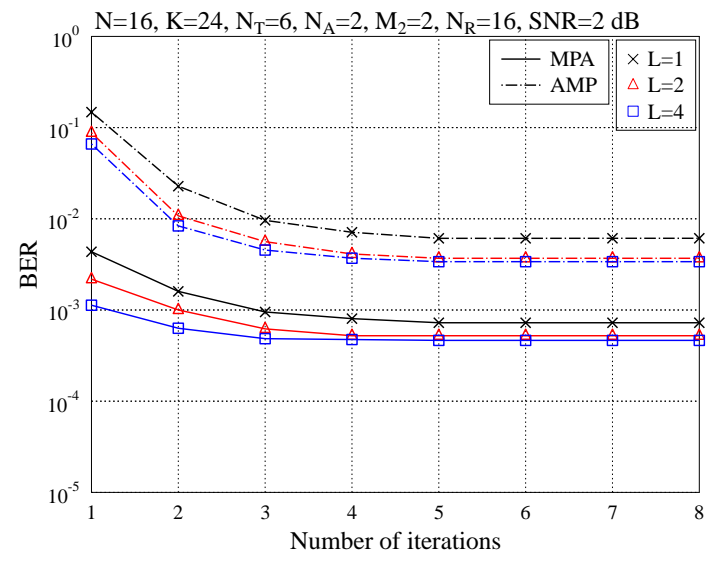

Fig. 11. BER vs. the number of iterations the $N_{R}=16$ STC/GSM-SCDMA systems employing MPA or AMP detection for supporting $K=24$ users by $N=16$ subcarriers at the SNR $\gamma=2 \mathrm{~dB}$, where each user is equipped with $N_{T}=4,6$ or 7 TAs, when communicating over the $L=4$ frequencyselective Rayleigh fading channel.

BER degradation, but extra bits may be conveyed. In Fig. 10, when $N_{A}$ is fixed to $2, N_{T}=4,6$ and 7 give $b_{1}=2,3$ and 4 bits to be conveyed by the activated TA indices per SD. By contrast, the increase of $M_{2}$ from 2 to 4 results in less than $0.5 \mathrm{~dB}$ SNR loss at a BER of $10^{-3}$, regardless of $N_{T} \mathrm{~s}$, while doubling the spectral efficiency.

Finally, Fig. 11 characterizes the convergence of the proposed MPA and low-complexity AMP detector for the $N_{R}=$ 16 STC/GSM-SCDMA systems at the SNR of $\gamma=2 \mathrm{~dB}$. The system parameters employed in Fig. 11 are the same as those adopted in Fig. 8. Since the BER always converges after the first 4 iterations for AMP detection and after the first 3 iterations for MPA detection, we fix the number of iterations to $I=\bar{I}=8$ in all simulations of the MPA or AMP detectors.

\section{B. Complexity}

In this section, the computational complexity per iteration of the proposed MPA and AMP detectors is quantified and compared in terms of the number of floating point operations (FLOPs). More specifically, we assume that

1) the multiplication of two real numbers requires a single FLOP;

2) the multiplication of a single complex number and a single real number requires 2 FLOPs;

3) the multiplication of a pair of complex numbers (excluding the conjugate multiplication) requires 6 FLOPs;

4) the multiplication of a complex number and its conjugate requires 3 FLOPs.

We also assume that the calculation of $\exp (\cdot)$ is carried by a look-up table [39].

First, as discussed in Section III-A, the complexity $\mathcal{C}_{\text {MPA }}$ of the MPA detector per iteration is comprised of the VN update of (12) and CN update of (16). The complexity of the MPA
TABLE III

THE NUMBER OF FLOPS REQUIRED FOR THE EQUATIONS EMPLOYED IN THE AMP DETECTION.

\begin{tabular}{c|l}
\hline Equation & FLOPs \\
\hline \hline$(29)$ & $C_{1}=4 N_{R} N Z N_{A} M_{1} M_{2} d_{x}$ \\
\hline$(30)$ & $C_{2}=N_{R} N Z N_{A} M_{1} d_{x}\left(6 M_{2}-1\right)$ \\
\hline$(23)$ & $C_{3}=N_{R} N Z\left[6 N_{A} M_{1}\left(d_{x}-1\right)+2\right]$ \\
\hline$(24)$ & $C_{4}=N_{R} N Z\left[N_{A} M_{1}\left(d_{x}-1\right)+1\right]$ \\
\hline$(27)$ & $C_{5}=K M_{1} N_{A} Z\left[N_{R}\left(d_{c}-1\right)+1\right]$ \\
\hline$(28)$ & $C_{6}=K M_{1} N_{A} Z\left[8 N_{R}\left(d_{c}-1\right)+1\right]$ \\
\hline$(26)$ & $C_{7}=K N_{R} d_{c} Z\left(11 N_{A} M_{1} M_{2}+1\right)$ \\
\hline
\end{tabular}

detection is already given in [46], which can be expressed as

$$
\begin{aligned}
\mathcal{C}_{\mathrm{MPA}}= & N_{R} N Z d_{x} \\
& \times\left[\left(7 d_{x}-1\right)\left(M_{1} M_{2}^{N_{A}}\right)^{d_{x}}+\left(d_{c}-1\right) M_{1} M_{2}^{N_{A}}\right] .
\end{aligned}
$$

By contrast, during each AMP operation, the number of FLOPs required to perform the operations of Lines 2-5 in Algorithm 1 is summarized in Table III. Hence, the complexity per iteration of the AMP detector in terms of FLOPs can be expressed as the sum of the expressions in Table III, which can be formulated as

$$
\mathcal{C}_{\mathrm{AMP}}=\sum_{j=1}^{7} C_{j} .
$$

Fig. 12 characterizes the computational complexity per iteration vs. the number of TAs at each user of the proposed $N_{A}=2$ STC/GSM-SCDMA systems employing MPA or AMP detection for supporting $K=16,24$ and 32 users by $N=16$ subcarriers, where $N_{R}=16$, when communicating over the frequency-selective Rayleigh fading channel having $L=4$, where (a) $M_{2}=2$ and (b) $M_{2}=4$. We can see from both Fig. 12(a) and Fig. 12(b) that the AMP detector requires a much lower number of FLOPs to complete a single iteration. For instance, when detecting a $N=16$ and $K=24$ STC/GSM-SCDMA system employing $N_{T}=6$ TAs, $N_{A}=2$ activated TAs and $N_{R}=16$ RAs, the AMP detection requires only $1.2 \times 10^{6}$ FLOPs to complete a single iteration, which is 1000 times lower than that of the MPA detector. Furthermore, we can see from Fig. 12(b) that in the case of $M_{2}=4$, when detecting the same $N=16$ and $K=24$ STC/GSMSCDMA system employing $N_{T}=6$ TAs, $N_{A}=2$ activated TAs and $N_{R}=16$ RAs, the complexity per iteration of the MPA detector is in excess of $10^{10}$, which is excessive for its practical implementation. By contrast, the complexity per iteration of detecting the same STC/GSM-SCDMA system using the AMP detector only linearly increases to $2.3 \times 10^{6}$ FLOPs, which readily facilitates the implementation of more complex STC/GSM-SCDMA systems.

Fig. 13 shows the total number of FLOPs vs. the number of TAs at each user of our STC/GSM-SCDMA systems employing either MPA or AMP detection using $I=8$ iterations with the same parameters as those in Fig. 12. Similar conclusions to those shown in Fig. 12 can be drawn, since the total number of FLOPs required for MPA and AMP are $\mathcal{C}_{\mathrm{AMP} \text {, total }}=I \sum_{j=1}^{7} C_{j}$ and $\mathcal{C}_{\mathrm{MPA} \text {, total }}=I N_{R} N Z d_{x}\left[\left(7 d_{x}-\right.\right.$ 1) $\left(M_{1} M_{2}^{N_{A}}\right)^{d_{x}}+\left(d_{c}-1\right) M_{1} M_{2}^{N_{A}}$, respectively. 


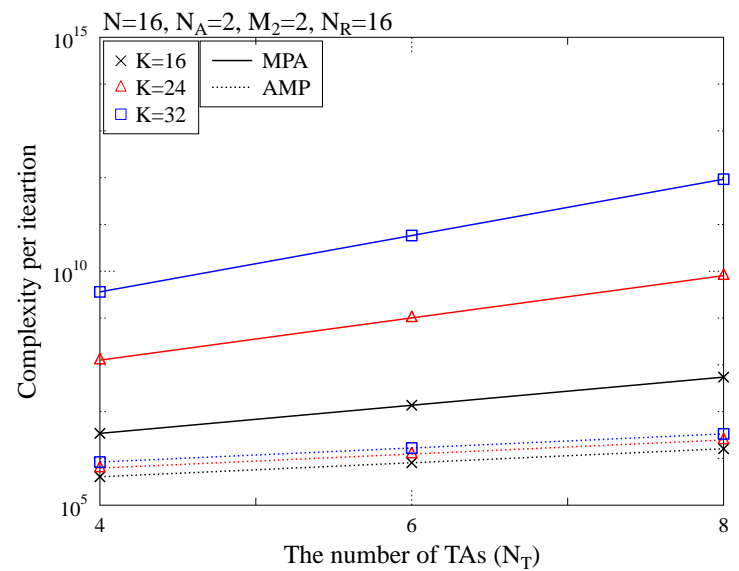

(a)

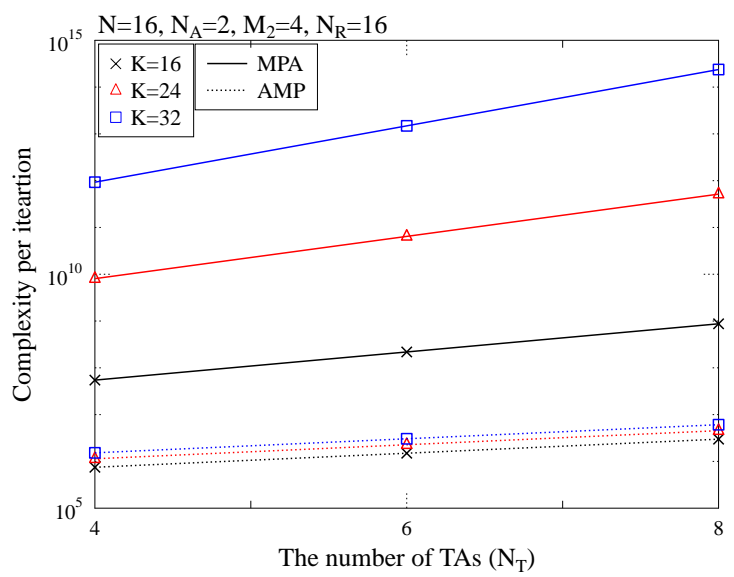

(b)

Fig. 12. Computational complexity per iteration vs. the number of TAs equipped at each user of the proposed $N_{A}=2, N_{R}=16 \mathrm{STC} / \mathrm{GSM}-\mathrm{SCDMA}$ systems employing MPA or AMP detection for supporting $K=16,24$ and 32 users by $N=16$ subcarriers, where (a) $M_{2}=2$ and (b) $M_{2}=4$.

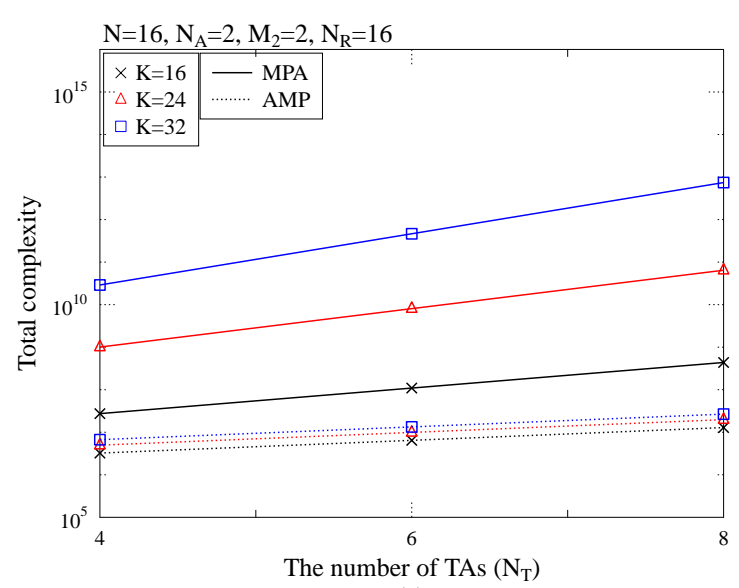

(a)

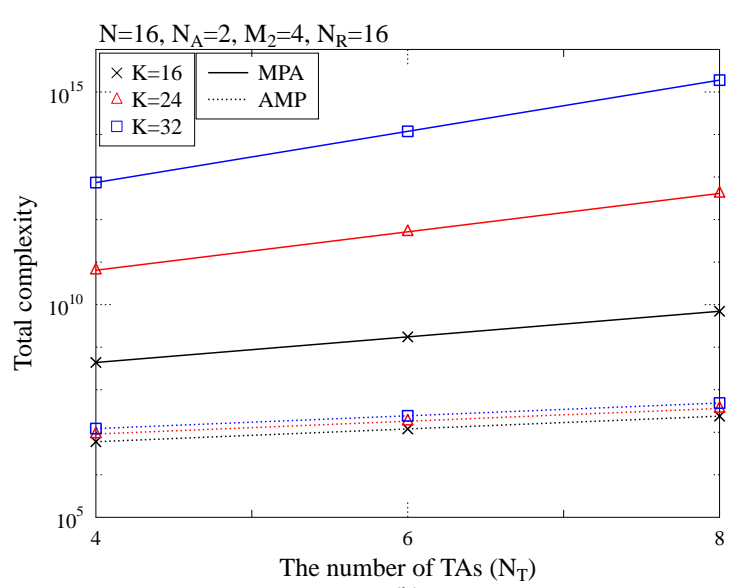

(b)

Fig. 13. Total computational complexity vs. the number of TAs equipped at each user of the proposed $N_{A}=2, N_{R}=16$ STC/GSM-SCDMA systems employing MPA or AMP detection with $I=8$ iterations for supporting $K=16,24$ and 32 users by $N=16$ subcarriers, where (a) $M_{2}=2$ and (b) $M_{2}=4$.

\section{CONCLUSIONS}

A STC/GSM-SCDMA scheme has been proposed, which benefits from transmit diversity in both the spatial and FD and provides a promising solution to the pervasive connectivity of the devices in the next-generation wireless communications. Furthermore, a single-user BER bound was derived as the benchmark of our STC/GSM-SCDMA system. Additionally, a pair of novel detectors has been conceived for our proposed STC/GSM-SCDMA system. In particular, the AMP detector has a 1000 times lower complexity than that of the MPA detector, at the cost of less than $2 \mathrm{~dB}$ SNR loss, when both are applied to our STC/GSM-SCDMA system having a normalized user load of $150 \%$. Our BER results also demonstrated that the proposed STC/GSM-SCDMA system outperforms the MIMO-NOMA schemes of $[31,33]$.

Our future work will investigate the theoretical multiuser performance of our proposed STC/GSM-SCDMA system. Furthermore, specifically low-complexity tailored AMP-assisted detector designs will be conceived for various MIMO-SCMA systems without significantly degrading the BER performance.
Another interesting idea to be investigated is the employment of partial Gaussian approximation-based MPA detection [47], which has the potential of further reducing the detection complexity of our STC/GSM-SCDMA scheme.

\section{REFERENCES}

[1] X. Chen, D. W. K. Ng, W. Yu, E. G. Larsson, N. Al-Dhahir, and R. Schober, "Massive access for $5 \mathrm{G}$ and beyond," arXiv preprint arXiv:2002.03491, 2020.

[2] S. Ali, N. Rajatheva, and W. Saad, "Fast uplink grant for machine type communications: Challenges and opportunities," IEEE Communications Magazine, vol. 57, no. 3, pp. 97-103, 2019.

[3] S. K. Sharma and X. Wang, "Toward massive machine type communications in ultra-dense cellular IoT networks: Current issues and machine learning-assisted solutions," IEEE Communications Surveys \& Tutorials, vol. 22, no. 1, pp. 426-471, 2019.

[4] Z. Xiang, W. Yang, Y. Cai, Z. Ding, Y. Song, and Y. Zou, "NOMA assisted secure short-packet communications in IoT," IEEE Wireless Communications, vol. 27, no. 4, pp. 8-15, 2020.

[5] Y. Liu, Z. Qin, Y. Cai, Y. Gao, G. Y. Li, and A. Nallanathan, "UAV communications based on non-orthogonal multiple access," IEEE Wireless Communications, vol. 26, no. 1, pp. 52-57, 2019.

[6] T. Hou, Y. Liu, Z. Song, X. Sun, and Y. Chen, "Exploiting NOMA for UAV communications in large-scale cellular networks," IEEE Transactions on Communications, vol. 67, no. 10, pp. 6897-6911, 2019. 
[7] T. Hou, Y. Liu, Z. Song, X. Sun, Y. Chen, L. Hanzo et al., "Reconfigurable intelligent surface aided NOMA networks," arXiv preprint arXiv:1912.10044, 2019.

[8] Z. Ding, R. Schober, P. Fan, and H. V. Poor, "OTFS-NOMA: An efficient approach for exploiting heterogenous user mobility profiles,' IEEE Transactions on Communications, vol. 67, no. 11, pp. 7950-7965, 2019.

[9] R. Hoshyar, F. P. Wathan, and R. Tafazolli, "Novel low-density signature for synchronous CDMA systems over AWGN channel," IEEE Transactions on Signal Processing, vol. 56, no. 4, pp. 1616-1626, 2008.

[10] R. Razavi, A.-I. Mohammed, M. A. Imran, R. Hoshyar, and D. Chen, "On receiver design for uplink low density signature OFDM (LDSOFDM)," IEEE Transactions on Communications, vol. 60, no. 11, pp 3499-3508, 2012.

[11] H. Nikopour and H. Baligh, "Sparse code multiple access," in 2013 IEEE 24th Annual International Symposium on Personal, Indoor, and Mobile Radio Communications (PIMRC). IEEE, 2013, pp. 332-336.

[12] Z. Ding, R. Schober, and H. V. Poor, "A general MIMO framework for NOMA downlink and uplink transmission based on signal alignment," IEEE Transactions on Wireless Communications, vol. 15, no. 6, pp. 4438-4454, 2016.

[13] Z. Yang, Z. Ding, P. Fan, and N. Al-Dhahir, "A general power allocation scheme to guarantee quality of service in downlink and uplink NOMA systems," IEEE Transactions on Wireless Communications, vol. 15 no. 11, pp. 7244-7257, 2016.

[14] Y. Liu, Z. Qin, M. Elkashlan, Z. Ding, A. Nallanathan, and L. Hanzo, "Nonorthogonal multiple access for 5G and beyond," Proceedings of the IEEE, vol. 105, no. 12, pp. 2347-2381, 2017.

[15] W. Yuan, N. Wu, A. Zhang, X. Huang, Y. Li, and L. Hanzo, "Iterative receiver design for FTN signaling aided sparse code multiple access," IEEE Transactions on Wireless Communications, vol. 19, no. 2, pp. 915928, 2019

[16] W. Yuan, N. Wu, Q. Guo, D. W. K. Ng, J. Yuan, and L. Hanzo, "Iterative joint channel estimation, user activity tracking, and data detection for FTN-NOMA systems supporting random access," IEEE Transactions on Communications, vol. 68, no. 5, pp. 2963-2977, 2020.

[17] W. Yuan, N. Wu, J. Yuan, D. W. K. Ng, and L. Hanzo, "Joint data and active user detection for grant-free FTN-NOMA in dynamic networks," arXiv preprint arXiv:2002.07992, 2020.

[18] M. Di Renzo, H. Haas, A. Ghrayeb, S. Sugiura, and L. Hanzo, "Spatial modulation for generalized MIMO: Challenges, opportunities, and implementation," Proceedings of the IEEE, vol. 102, no. 1, pp. 56-103, 2014.

[19] J. Wang, S. Jia, and J. Song, "Generalised spatial modulation system with multiple active transmit antennas and low complexity detection scheme," IEEE Transactions on Wireless Communications, vol. 11, no. 4 pp. 1605-1615, 2012.

[20] Y. Bian, X. Cheng, M. Wen, L. Yang, H. V. Poor, and B. Jiao, "Differential spatial modulation," IEEE Transactions on Vehicular Technology, vol. 64 , no. 7 , pp. 3262-3268, 2014

[21] R. Mesleh, S. S. Ikki, and H. M. Aggoune, "Quadrature spatial modulation," IEEE Transactions on Vehicular Technology, vol. 64, no. 6, pp. 2738-2742, 2014.

[22] A. Younis, R. Mesleh, and H. Haas, "Quadrature spatial modulation performance over Nakagami- $m$ fading channels," IEEE Transactions on Vehicular Technology, vol. 65, no. 12, pp. 10227-10231, 2015.

[23] N. Ishikawa and S. Sugiura, "Rectangular differential spatial modulation for open-loop noncoherent massive-MIMO downlink," IEEE Transactions on Wireless Communications, vol. 16, no. 3, pp. 1908-1920, 2017.

[24] E. Basar, U. Aygolu, E. Panayirci, and H. V. Poor, "Space-time block coded spatial modulation," IEEE Transactions on Communications, vol. 59, no. 3, pp. 823-832, 2010.

[25] L. Xiao, Y. Xiao, C. Xu, X. Lei, P. Yang, S. Li, and L. Hanzo, "Compressed-sensing assisted spatial multiplexing aided spatial modulation," IEEE Transactions on Wireless Communications, vol. 17, no. 2 pp. 794-807, 2017.

[26] M. Di Renzo and H. Haas, "Bit error probability of space-shift keying MIMO over multiple-access independent fading channels," IEEE Transactions on Vehicular Technology, vol. 60, no. 8, pp. 3694-3711, 2011.

[27] T. L. Narasimhan, P. Raviteja, and A. Chockalingam, "Generalized spatial modulation in large-scale multiuser MIMO systems," IEEE Transactions on Wireless Communications, vol. 14, no. 7, pp. 3764 3779, 2015.

[28] T. Wang, S. Liu, F. Yang, J. Wang, J. Song, and Z. Han, "Generalized spatial modulation-based multi-user and signal detection scheme for terrestrial return channel with NOMA," IEEE Transactions on Broadcasting, vol. 64, no. 2, pp. 211-219, 2017
[29] Y. Liu, L.-L. Yang, and L. Hanzo, "Spatial modulation aided sparse code-division multiple access," IEEE Transactions on Wireless Communications, vol. 17, no. 3, pp. 1474-1487, 2018.

[30] M. Toka and O. Kucur, "Non-orthogonal multiple access with Alamouti space-time block coding," IEEE Communications Letters, vol. 22, no. 9 , pp. 1954-1957, 2018.

[31] Z. Pan, W. Liu, J. Lei, J. Luo, L. Wen, and C. Tang, "Multi-dimensional space-time block coding aided downlink MIMO-SCMA," IEEE Transactions on Vehicular Technology, vol. 68, no. 7, pp. 6657-6669, 2019.

[32] J. Dai, K. Niu, and J. Lin, "Iterative Gaussian-approximated message passing receiver for MIMO-SCMA system," IEEE Journal of Selected Topics in Signal Processing, vol. 13, no. 3, pp. 753-765, 2019.

[33] Y. Liu, L.-L. Yang, P. Xiao, H. Haas, and L. Hanzo, "Spatial modulated multicarrier sparse code-division multiple access," IEEE Transactions on Wireless Communications, vol. 19, no. 1, pp. 610-623, 2020.

[34] Y. Liu, L.-L. Yang, and L. Hanzo, "Sparse space-time-frequency-domain spreading for large-scale non-orthogonal multiple access," IEEE Transactions on Vehicular Technology, vol. 69, no. 10, pp. 12327-12332, 2020.

[35] _ " "Joint user-activity and data detection for grant-free spatialmodulated multi-carrier non-orthogonal multiple access," IEEE Transactions on Vehicular Technology, vol. 69, no. 10, pp. 11673-11684, 2020.

[36] S. M. Alamouti, "A simple transmit diversity technique for wireless communications," IEEE Journal on Selected Areas in Communications, vol. 16 , no. 8 , pp. 1451-1458, 1998 .

[37] L.-L. Yang, Multicarrier Communications. Chichester, United Kingdom: John Wiley, 2009.

[38] D. L. Donoho, A. Maleki, and A. Montanari, "Message-passing algorithms for compressed sensing," Proceedings of the National Academy of Sciences, vol. 106, no. 45, pp. 18914-18919, 2009.

[39] S. Wu, L. Kuang, Z. Ni, J. Lu, D. Huang, and Q. Guo, "Low-complexity iterative detection for large-scale multiuser MIMO-OFDM systems using approximate message passing," IEEE Journal of Selected Topics in Signal Processing, vol. 8, no. 5, pp. 902-915, 2014.

[40] J. P. Vila and P. Schniter, "Expectation-maximization Gaussian-mixture approximate message passing," IEEE Transactions on Signal Processing, vol. 61, no. 19, pp. 4658-4672, 2013.

[41] T. Van Erven and P. Harremos, "Rényi divergence and Kullback-Leibler divergence," IEEE Transactions on Information Theory, vol. 60, no. 7, pp. 3797-3820, 2014.

[42] M. K. Simon and M.-S. Alouini, Digital Communication over Fading Channels, 2nd ed. New York: John Wiley \& Sons, 2005.

[43] T. A. Lamahewa, M. K. Simon, R. A. Kennedy, and T. D. Abhayapala, "Performance analysis of space-time codes in realistic propagation environments: A moment generating function-based approach," Journal of Communications and Networks, vol. 7, no. 4, pp. 450-461, 2005.

[44] L. Li, Z. Ma, P. Z. Fan, and L. Hanzo, "High-dimensional codebook design for the SCMA down link," IEEE Transactions on Vehicular Technology, vol. 67, no. 10, pp. 10118-10 122, 2018.

[45] Y. Wu, S. Zhang, and Y. Chen, "Iterative multiuser receiver in sparse code multiple access systems," in 2015 IEEE International Conference on Communications (ICC). IEEE, 2015, pp. 2918-2923.

[46] Z. Tang, J. Wang, J. Wang, and J. Song, "A low-complexity detection algorithm for uplink NOMA system based on Gaussian approximation," in 2017 IEEE Wireless Communications and Networking Conference (WCNC). IEEE, 2017, pp. 1-6.

[47] W. Yuan, N. Wu, Q. Guo, Y. Li, C. Xing, and J. Kuang, "Iterative receivers for downlink MIMO-SCMA: Message passing and distributed cooperative detection," IEEE Transactions on Wireless Communications, vol. 17 , no. 5 , pp. $3444-3458,2018$ 